Draft Version November 4, 2018

Preprint typeset using $\mathrm{LAT}_{\mathrm{E}} \mathrm{X}$ style emulateapj v. 08/22/09

\title{
MEASUREMENTS OF SECONDARY COSMIC MICROWAVE BACKGROUND ANISOTROPIES WITH THE SOUTH POLE TELESCOPE
}

M. Lueker, ${ }^{1}$ C. L. Reichardt,${ }^{1}$ K. K. Schaffer, ${ }^{2,3}$ O. Zahn, ${ }^{4}$ P. A. R. Ade, ${ }^{5}$ K. A. Aird, ${ }^{6}$ B. A. Benson, ${ }^{1,2,3}$ L. E. Bleem ${ }^{2,7}$ J. E. Carlstrom, ${ }^{2,3,7,8}$ C. L. Chang, ${ }^{2,3}$ H.-M. Cho, ${ }^{1}$ T. M. Crawford ${ }^{2,8}$ A. T. Crites, ${ }^{2,8}$ T. De HaAn, ${ }^{9}$ M. A. Dobbs,${ }^{9}$ E. M. George, ${ }^{1}$ N. R. Hall,${ }^{10}$ N. W. Halverson, ${ }^{11}$ G. P. Holder,${ }^{9}$ W. L. Holzapfel, ${ }^{1}$ J. D. Hrubes ${ }^{6}$ M. Joy, ${ }^{12}$ R. Keisler,${ }^{2,7}$ L. Knox, ${ }^{10}$ A. T. Lee, ${ }^{1,13}$ E. M. Leitch, ${ }^{2,8}$ J. J. McMahon, ${ }^{2,3,14}$ J. Mehl, ${ }^{1}$ S. S. Meyer, ${ }^{2,3,7,8}$ J. J. Mohr $,{ }^{15}, 16,17$ T. E. Montroy ${ }^{18}$ S. Padin, ${ }^{2,8}$ T. Plagge, ${ }^{1}$ C. Pryke, $,{ }^{2,3}, 8$ J. E. Ruhl ${ }^{18}$ L. Shaw, ${ }^{9,19}$ E. Shirokoff ${ }^{1}$ H. G. Spieler, ${ }^{13}$ Z. Staniszewski, ${ }^{18}$ A. A. Stark, ${ }^{20}$ K. Vanderlinde, ${ }^{9}$ J. D. Vieira, ${ }^{2,7}$ And R. Williamson ${ }^{2,8}$ Draft version November 4, 2018

\begin{abstract}
We report cosmic microwave background (CMB) power spectrum measurements from the first $100 \mathrm{deg}^{2}$ field observed by the South Pole Telescope (SPT) at 150 and $220 \mathrm{GHz}$. On angular scales where the primary CMB anisotropy is dominant, $\ell \lesssim 3000$, the SPT power spectrum is consistent with the standard $\Lambda$ CDM cosmology. On smaller scales, we see strong evidence for a point source contribution, consistent with a population of dusty, star-forming galaxies. After we mask bright point sources, anisotropy power on angular scales of $3000<\ell<9500$ is detected with a signal-to-noise $\gtrsim 50$ at both frequencies. We combine the 150 and $220 \mathrm{GHz}$ data to remove the majority of the point source power, and use the point source subtracted spectrum to detect Sunyaev-Zel'dovich (SZ) power at $2.6 \sigma$. At $\ell=3000$, the $\mathrm{SZ}$ power in the subtracted bandpowers is $4.2 \pm 1.5 \mu \mathrm{K}^{2}$, which is significantly lower than the power predicted by a fiducial model using WMAP 5 cosmological parameters. This discrepancy may suggest that contemporary galaxy cluster models overestimate the thermal pressure of intracluster gas. Alternatively, this result can be interpreted as evidence for lower values of $\sigma_{8}$. When combined with an estimate of the kinetic SZ contribution, the measured SZ amplitude shifts $\sigma_{8}$ from the primary CMB anisotropy derived constraint of $0.794 \pm 0.028$ down to $0.773 \pm 0.025$. The uncertainty in the constraint on $\sigma_{8}$ from this analysis is dominated by uncertainties in the theoretical modeling required to predict the amplitude of the SZ power spectrum for a given set of cosmological parameters.

Subject headings: cosmology - cosmology:cosmic microwave background - cosmology: observations large-scale structure of universe
\end{abstract}

Electronic address: lueker@socrates.berkeley.edu

${ }^{1}$ Department of Physics, University of California, Berkeley, CA 94720

${ }^{2}$ Kavli Institute for Cosmological Physics, University of Chicago, 5640 South Ellis Avenue, Chicago, IL 60637

${ }^{3}$ Enrico Fermi Institute, University of Chicago, 5640 South Ellis Avenue, Chicago, IL 60637

4 Berkeley Center for Cosmological Physics, Department of Physics, University of California, and Lawrence Berkeley National Labs, Berkeley, CA 94720

5 Department of Physics and Astronomy, Cardiff University, CF24 3YB, UK

${ }^{6}$ University of Chicago, 5640 South Ellis Avenue, Chicago, IL 60637

${ }^{7}$ Department of Physics, University of Chicago, 5640 South Ellis Avenue, Chicago, IL 60637

8 Department of Astronomy and Astrophysics, University of Chicago, 5640 South Ellis Avenue, Chicago, IL 60637

${ }^{9}$ Department of Physics, McGill University, 3600 Rue University, Montreal, Quebec H3A 2T8, Canada

10 Department of Physics, University of California, One Shields Avenue, Davis, CA 95616

${ }^{11}$ Department of Astrophysical and Planetary Sciences and Department of Physics, University of Colorado, Boulder, CO 80309

12 Department of Space Science, VP62, NASA Marshall Space Flight Center, Huntsville, AL 35812

${ }^{13}$ Physics Division, Lawrence Berkeley National Laboratory, Berkeley, CA 94720

${ }^{14}$ Department of Physics, University of Michigan, 450 Church Street, Ann Arbor, MI, 48109

${ }^{15}$ Department of Physics, Ludwig-Maximilians-Universität, Scheinerstr. 1, 81679 München, Germany

16 Excellence Cluster Universe, Boltzmannstr. 2, 85748 Garching, Germany

\section{INTRODUCTION}

Anisotropy in the cosmic microwave background (CMB) has been well-characterized on angular scales larger than a few arcminutes (Jones et al. 2006: Reichardt et al. 2009a; Nolta et al. 2009 Brown et al. 2009), providing stringent tests of cosmological theory and strong evidence for the $\Lambda \mathrm{CDM}$ cosmological model (Hinshaw et al. 2009, Komatsu et al. 2009). The primary CMB anisotropy is a direct record of density fluctuations at the surface of last scattering. On angular scales $\lesssim 10$ arcminutes, the primary CMB anisotropy is exponentially damped due to photon diffusion in the primordial plasma (Silk 1968); the resulting decline in power with increasing multipole is known as the "damping tail". The anisotropy on very small scales, which is only beginning to be explored experimentally, is instead dominated by foreground emission and secondary distortions to the CMB introduced after the surface of last scattering. This secondary CMB anisotropy is produced by interactions

\footnotetext{
17 Max-Planck-Institut für extraterrestrische Physik, Giessenbachstr. 85748 Garching, Germany

${ }^{18}$ Physics Department, Center for Education and Research in Cosmology and Astrophysics, Case Western Reserve University, Cleveland, OH 44106

${ }^{19}$ Department of Physics, Yale University, P.O. Box 208210, New Haven, CT 06520-8120

${ }^{20}$ Harvard-Smithsonian Center for Astrophysics, 60 Garden Street, Cambridge, MA 02138
} 
of CMB photons with large scale structure and can potentially provide sensitive and independent tests of cosmological parameters important to structure formation.

The most significant source of secondary CMB temperature anisotropy on small angular scales is predicted to be the inverse Compton scattering of CMB photons by the hot plasma gravitationally bound to massive, collapsed objects (Sunyaev \& Zeldovich 1972). This is known as the thermal Sunyaev-Zel'dovich (tSZ) effect. The tSZ effect distorts the blackbody spectrum of the primary $\mathrm{CMB}$, creating a decrement in intensity at low frequencies and an increment at high frequencies with a null near $220 \mathrm{GHz}$. At $150 \mathrm{GHz}$, the anisotropy induced by the tSZ effect is expected to dominate over the primary CMB fluctuations at multipoles $\ell \gtrsim 3000$. The power contributed to CMB maps by the tSZ effect depends sensitively on the normalization of the matter power spectrum, as parametrized by the RMS of the mass distribution on $8 \mathrm{~h}^{-1} \mathrm{Mpc}$ scales, $\sigma_{8}$.

Galaxy clusters over a wide range of mass and redshift contribute significantly to the tSZ power spectrum. It is challenging to accurately model the low-mass or highredshift clusters. For instance, lower mass clusters are more sensitive to non-gravitational heating effects such as by AGN, while we have limited observational data on high-redshift clusters. There is considerable uncertainty as to the expected shape and amplitude of the tSZ power spectrum due to the current lack of knowledge of the properties of intracluster gas in low mass and high redshift galaxy clusters.

The scattered CMB photons also obtain a net Doppler shift when ionized matter is moving with respect to the rest frame of the CMB. This so-called kinetic SunyaevZel'dovich (kSZ) effect (Sunyaev \& Zeldovich 1980) depends solely on the motion and density of free electrons. In contrast to the tSZ effect, the $\mathrm{kSZ}$ effect has contributions from electrons with temperatures as low as $10^{4} \mathrm{~K}$. Therefore higher-redshift epochs, before massive objects finish collapsing, are expected to have relatively larger contributions to the kSZ power. Recent simulations and analytic models also predict a sizable signal from the epoch of the first radiative sources which form ionized regions several tens of Mpc across, within a largely neutral Universe. Low-redshift galaxy clusters dominate the power on small angular scales, while high-redshift reionizing regions have their largest relative contribution on angular scales around $\ell=2000$. At $150 \mathrm{GHz}$, the kinetic effect is expected to amount to tens of percent of the total SZ power.

Only a handful of experiments have had sufficient sensitivity and angular resolution to probe the damping tail of the CMB anisotropy. Early measurements at $30 \mathrm{GHz}$ by CBI (Mason et al. 2003. Bond et al. 2005) reported a $>3 \sigma$ excess above the expected CMB power at multipoles of $\ell>2000$. Observations with the BIMA array at $30 \mathrm{GHz}$ (Dawson et al. 2006) also reported a nearly $2 \sigma$ detection of excess power at $\ell=5237$. However, more recently, the SZA experiment (also observing at $30 \mathrm{GHz}$ ) has published an upper limit of $149 \mu \mathrm{K}^{2}$ at $95 \%$ confidence on excess power at these multipoles (Sharp et al. 2009) in apparent conflict with the previous CBI and BIMA results. For the relatively small patch $\left(0.1 \mathrm{deg}^{2}\right)$ observed by BIMA, the non-Gaussian nature of the SZ sky means that there is no significant tension between the BIMA and SZA results. The latest CBI measurements (Sievers et al. 2009) include more data, improved radio source removal, and a proper treatment of nonGaussianity of the SZ sky. These measurements continue to suggest excess power but with a significance of only $1.6 \sigma$.

At $150 \mathrm{GHz}$, the ACBAR (Reichardt et al. 2009a) and QUaD (Friedman et al. 2009) experiments have both measured the damping tail of the primary CMB anisotropy at $\ell<3000$ with high signal to noise. Either with or without the addition of the expected foreground and tSZ contributions, the power measured at the highest multipoles by both experiments is consistent with primary CMB anisotropy. In the last year, the results of $150 \mathrm{GHz}$ observations out to $\ell=10000$ made with the Bolocam (Sayers et al. 2009) and APEX-SZ (Reichardt et al. 2009b) experiments have been released. These experiments have been used to place upper limits on power above the primary $\mathrm{CMB}$ of $1080 \mu \mathrm{K}^{2}$ and $105 \mu \mathrm{K}^{2}$ respectively at $95 \%$ confidence. The constraints on $\sigma_{8}$ from these upper limits remain weak, in no small part due to the large, highly non-Gaussian sample variance of the tSZ effect on the small $\sim 1 \mathrm{deg}^{2}$ patches of sky observed by Bolocam and APEX-SZ. The cosmic variance of the tSZ effect will be significantly reduced in the on-going $\gtrsim 100 \mathrm{deg}^{2}$ surveys being conducted by next-generation experiments such as ACT (?) and SPT.

In addition to the tSZ and kSZ effects, foreground emission is important on these small angular scales. After bright radio sources are removed, the most significant foreground at 150 and $220 \mathrm{GHz}$ is expected to be a population of unresolved, faint, dusty, star-forming galaxies (DSFGs) with a rest frame emission spectrum that peaks in the far infrared. These sources have been studied at higher frequencies close to the peak of their emission spectrum ${ }^{21}$ (e.g. Holland et al. (1999); Kreysa et al. (1998); Glenn et al. (1998); Viero et al. (2009)), however extrapolating their fluxes to $150 \mathrm{GHz}$ remains uncertain. Adding to the challenge is the expected significant clustering of these sources (Haiman \& Knox 2000; Knox et al. 2001; Righi et al. 2008; Sehgal et al. 2009). IR emission from clustered DSFGs was first observed with the Spitzer telescope at $160 \mu \mathrm{m}$ (Lagache et al. 2007) and more recently this clustering has also been observed at sub-mm wavelengths by the BLAST experiment (Viero et al. 2009). The clustering of these DSFGs is expected to produce anisotropic power at $150 \mathrm{GHz}$ with an angular power spectrum that is similar to that of the SZ effect. However, emission from DSFGs is spectrally separable from the SZ effect and the SZ power spectrum can be recovered by combining information from overlapping maps at 150 and $220 \mathrm{GHz}$.

In this work, we present measurements by the South Pole Telescope (SPT) which comprise the first significant detections of anisotropy power for $\ell>3000$ at 150 and $220 \mathrm{GHz}$. The SPT has sufficient angular resolution, sensitivity and sky coverage to produce high-precision measurements of anisotropy over a range of multipoles from $\sim 100<\ell<9500$. However, for the immediate goal

21 Since these sources are typically brightest at sub-millimeter wavelengths they are also referred to in the literature as submillimeter galaxies (SMGs). 
of measuring secondary CMB anisotropies, we start with the first bandpower at $\ell=2000$ where primary CMB still dominates the power spectrum. We combine bandpowers from two frequencies to minimize the DSFG contribution and produce the first significant detection of the SZ contribution to the CMB power spectrum. In a companion paper Hall et al. (2009, hereafter H09), the SPT power spectra are used to place constraints on the amplitude of the Poisson and clustered components of DSFG power. This is the first detection of the clustered DSFG power at mm-wavelengths. We use three results from H09 in setting limits on the residual point source power in the DSFG-subtracted bandpowers (\$6.2): an argument that residual clustered components are negligible, a measurement of the Poisson point source power at $150 \mathrm{GHz}$, and a limit on the scatter in spectral index between DSFGs.

We describe the instrument, observations, beams, and calibration strategy in $\$ 2$. The time-ordered data (TOD) filtering and map-making algorithm is outlined in $\$ 3$. along with the procedure to derive bandpowers from maps. The results of tests for systematic errors applied to the SPT data are discussed in \$4 and the expected astronomical foregrounds are described in $\$ 5$. The bandpowers and cosmological interpretation are given in 86 .

\section{INSTRUMENT AND OBSERVATIONS}

The SPT is an off-axis Gregorian telescope with a $10-\mathrm{m}$ diameter primary mirror located at the South Pole. The telescope is optimized to perform high resolution surveys of low surface brightness sources. The first receiver to be placed on the SPT has the primary goal of identifying a mass-limited sample of galaxy clusters, the first of which were reported in Staniszewski et al. 2009, hereafter S09). The receiver is equipped with a 960-element array of superconducting transition edge sensor bolometers. The detectors are split between three frequency bands centered at $95 \mathrm{GHz}, 150 \mathrm{GHz}$, and $220 \mathrm{GHz}$, allowing the separation of the tSZ effect from the primary CMB anisotropy and foregrounds. Further details of the receiver and telescope can be found in Ruhl et al. (2004), Padin et al. (2008) and Carlstrom et al. (2009).

In this work, we use data at 150 and $220 \mathrm{GHz}$ from one $100 \mathrm{deg}^{2}$ field centered at right ascension $5^{\mathrm{h}} 30^{\mathrm{m}}$, declination $-55^{\circ}$ (J2000) observed by SPT in the first half of the 2008 austral winter. Data from SPT's third (95 $\mathrm{GHz}$ ) frequency band was unusable in 2008. The location of the field was chosen for overlap with the Blanco Cosmology Survey (BCS) 22 optical survey and low dust emission. We observed this field for a total of 779 hours, of which 575 hours is used in the analysis after passing data quality cuts. The final map noise is $18 \mu \mathrm{K}$-arcmin ${ }^{23}$ at $150 \mathrm{GHz}$ and $40 \mu \mathrm{K}$-arcmin at $220 \mathrm{GHz}$. This field accounts for half the sky area observed in 2008 and one eighth of the total area observed by SPT to date.

The scanning strategy used for these observations involves constant-elevation scans across the $10^{\circ}$ wide field. After each scan back and forth across the field, the telescope executes a $0.125^{\circ}$ step in elevation. A complete

\footnotetext{
22 http://cosmology.illinois.edu/BCS

23 Throughout this work, the unit $\mathrm{K}$ refers to equivalent fluctuations in the CMB temperature, i.e., the level of temperature fluctuation of a $2.73 \mathrm{~K}$ blackbody that would be required to produce the same power fluctuation. The conversion factor is given by the derivative of the blackbody spectrum, $\frac{d B}{d T}$, evaluated at $2.73 \mathrm{~K}$.
}

set of scans covering the entire field takes approximately two hours, and we refer to each complete set as an observation. Successive observations use a series of different starting elevations to ensure even coverage of the field. Of the 300 observations used in this analysis, half were performed at an azimuth scanning speed of $0.44^{\circ}$ per second and half at a speed of $0.48^{\circ}$ per second.

\subsection{Beam Measurements}

The SPT beams are measured by combining maps of three sources: Jupiter, Venus, and the brightest point source in the $100 \mathrm{deg}^{2}$ field. Observations of Jupiter are used to measure a diffuse, low-level sidelobe in the range $15^{\prime}<\mathrm{r}<40^{\prime}$, where $\mathrm{r}$ is the radius to the beam center. Although this sidelobe has a low amplitude $(-50 \mathrm{~dB}$ at $\mathrm{r}=30^{\prime}$ ), it contains approximately $15 \%$ of the total beam solid angle. A measurement of this sidelobe is necessary for the cross-calibration with WMAP described in $\$ 2.2$. The observations of Jupiter show signs of potential nonlinearity in the response of the detectors for $\mathrm{r}<10^{\prime}$. For this reason we only use the observations of Jupiter to map the sidelobe at $\mathrm{r}>15^{\prime}$. Observations of Venus are used to measure the beam in the region $4^{\prime}<\mathrm{r}<15^{\prime}$. The angular extent of Jupiter or Venus has a negligible effect on the measurement of the relatively smooth beam features present at these large radii. The brightest point source in the map of the $100 \mathrm{deg}^{2}$ field is used to measure the beam within a radius of $4^{\prime}$. In this way, the random error in the pointing reconstruction ( $\left.7^{\prime \prime} \mathrm{RMS}\right)$ and its impact on the effective beam are taken into account. The pointing error has a negligible effect on the relatively smooth outer $\left(\mathrm{r}>4^{\prime}\right)$ region of the beam.

A composite beam map, $B(\theta, \phi)$, is assembled by merging maps of Jupiter, Venus, and the bright point source. The TOD are filtered prior to making these maps, in order to remove large-scale atmospheric noise. Masks with radii of $40^{\prime}, 25^{\prime}$, and $5^{\prime}$ are applied around the locations of Jupiter, Venus, and the point source, respectively. These masks ensure that the beam measurements are not affected by the filtering.

Using the the flat-sky approximation, we calculate the Fourier transform of the composite beam map, $B\left(\ell, \phi_{\ell}\right)$. From this, we compute the azimuthally-averaged beam function,

$$
B_{\ell}=\operatorname{Re}\left\{\frac{1}{2 \pi} \int \mathrm{B}\left(\ell, \phi_{\ell}\right) \mathrm{d} \phi_{\ell}\right\}
$$

We note that averaging $\left|B\left(\ell, \phi_{\ell}\right)\right|^{2}$ instead of $B\left(\ell, \phi_{\ell}\right)$ would result in a percent-level noise bias in $B_{\ell}$ at very high multipoles due to the presence of noise. The results in this work assume an axially symmetric beam, which is only an approximation for SPT. We simulate the effects of ignoring the asymmetry on the bandpowers and find that the errors introduced by making this assumption are negligible.

Although the measured beam function $B_{\ell}$ is used for the bandpower estimation, an empirical fit is used to quantify the errors on $B_{\ell}$. $B_{\ell}$ is fit to the empirical model

$$
B_{\ell}=a e^{-\frac{1}{2}\left(\sigma_{b} \ell\right)^{1.5}}+(1-a) e^{-\frac{1}{2}(0.00292 * \ell)^{1.8}} .
$$

There are two components: a main lobe (first term) and a diffuse shelf (second term). The form of the model and the numerical values of the slopes of the exponents 


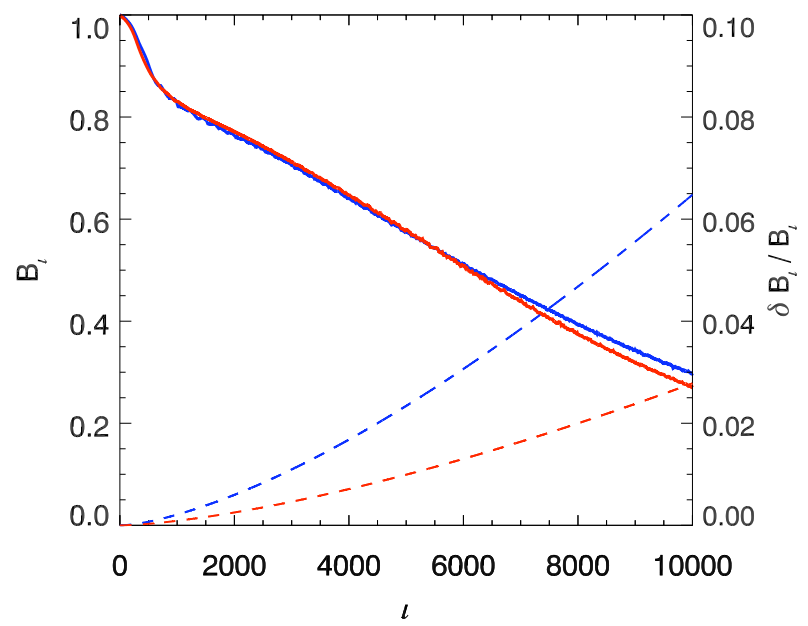

FIG. 1.- Average beam functions and uncertainties for SPT. Left axis: The SPT beam function for $150 \mathrm{GHz}$ (red) and $220 \mathrm{GHz}$ (blue). Right axis: The $1 \sigma$ uncertainties on the beam function for each frequency. The beam uncertainties shown here include only uncertainties on the main lobe beam width, $\sigma_{b}$, since the uncertainty of the sidelobe amplitude has been subsumed into the calibration uncertainty.

were constructed to provide a good fit to the measured $B_{\ell}$. We note that $B_{\ell}^{150}$ and $B_{\ell}^{220}$ are measured and fitted separately. The RMS difference between the model and measured $B_{\ell} \mathrm{s}$ is approximately $1 \%$. Two parameters remain free: $\sigma_{b}$, which describes the width of the main lobe, and $a$, which sets the relative normalization between the main lobe and the diffuse shelf. These parameters are left free to quantify the uncertainty in $B_{\ell}$. The uncertainty in the values of these parameters directly translates to an uncertainty in $B_{\ell}$.

There are a number of factors that limit the accuracy of the measurement of $B_{\ell}$. These include residual map noise, errors associated with the map-merging process, and spectral differences between the CMB and the sources used to measure the beam. The final uncertainties in the beam model parameters $\sigma_{b}$ and $a$ are constructed as the quadrature sum of the estimated uncertainties due to each of these individual sources of error. To a good approximation, the uncertainties on $\sigma_{b}$ and $a$ can be taken to be Gaussian and uncorrelated.

In practice, a change in the value of $a$ is equivalent to a change in the overall calibration for $\ell>700$. After the beam uncertainties are estimated, the uncertainty in $a$ is folded into the estimated uncertainty on the absolute calibration and the parameter $a$ is fixed to the best-fit value of 0.85 . The quoted beam uncertainties in the CosmoMC ${ }^{24}$ (Lewis \& Bridle 2002) data files in $\$ 6.1$ include only the uncertainty on $\sigma_{b}$. Figure 1 shows the measured beam functions for 150 and $220 \mathrm{GHz}$, along with the $1 \sigma$ uncertainties in the main lobe beam width $\sigma_{b}$.

\subsection{Calibration}

The calibration of the SPT data is tied to the superb WMAP5 absolute calibration through a direct comparison of $150 \mathrm{GHz}$ SPT maps with WMAP5 V and W-band

\footnotetext{
24 http://cosmologist.info/cosmomc
}

(61 and $94 \mathrm{GHz}$ ) maps (Hinshaw et al. 2009) of the same sky regions. The WMÁP5 maps are resampled according to the SPT pointing information, and the resulting TOD are passed through the SPT analysis pipeline to capture the effects of TOD filtering. The ratio of the cross-spectra of the filtered WMAP and SPT maps after correcting for the instrumental beams,

$$
c=\frac{\left\langle a_{\ell m, W M A P_{i}}^{*} a_{\ell m, W M A P_{j}}\right\rangle}{\left\langle a_{\ell m, S P T}^{*} a_{\ell m, W M A P_{j}}\left(\frac{B_{\ell}^{W M A P_{i}}}{B_{\ell}^{S P T}}\right)\right\rangle},
$$

is used to estimate the relative calibration factor between the two experiments. A similar procedure was used to calibrate the Boomerang, ACBAR, and QUaD experiments (Jones et al. 2006, Reichardt et al. 2009a: Brown et al. 2009). Dedicated SP' calibration scans of four large fields totaling $1250 \mathrm{deg}^{2}$ of sky were obtained during 2008. The results for these four fields are combined to achieve an absolute temperature calibration uncertainty of $3.6 \%$ at $150 \mathrm{GHz}$.

The $150 \mathrm{GHz}$ calibration is transferred to $220 \mathrm{GHz}$ through the overlapping coverage of SPT's high S/N maps. We calculate the relative calibration by examining the ratio of the cross-spectra between the 150 and $220 \mathrm{GHz}$ maps to the auto-spectra of the $150 \mathrm{GHz}$ map after correcting for the beam and filtering differences. We estimate the relative calibration uncertainty to be $6.2 \%$ and the final absolute calibration uncertainty of the $220 \mathrm{GHz}$ temperature map to be $7.2 \%$.

\section{ANALYSIS}

In this section we describe the pipeline used to process the TOD to maps, and from maps to bandpowers. Given the small sky area analyzed here, we use the flat-sky approximation. We generate maps in a flat-sky projection and analyze these maps using Fourier transforms. Thus, the discussion of filtering and data-processing techniques refer to particular modes by their corresponding angular wavenumber $\mathbf{k}$ in radians. Note that in these units $|\mathbf{k}|=\ell$.

\subsection{Map-making}

We generate one map per frequency band for each twohour observation of the field. The map-making pipeline used for this analysis is very similar to that used by S09, with some small modifications. An overview of the pipeline is provided below, emphasizing the aspects of data processing and map-making that are most important for understanding the power spectrum analysis.

\subsubsection{Data Selection}

Variations in observing conditions and daily receiver setup can affect the performance of individual bolometer channels. The first step in the data processing is to select the set of bolometers with good performance for each individual observation. The criteria for selecting these detectors are primarily based on responsivity (determined through the series of calibrations performed prior to each field observation) and noise performance. Detectors are rejected if they have a low signal-to-noise response to a chopped thermal calibration source or atmospheric emission (modulated through a short elevation scan). They 
are also rejected if their noise power spectrum is heavily contaminated by readout-related line-like features. More detail on the bolometer cuts can be found in S09. In addition, bolometers that have a responsivity or inversenoise-based weight more than a factor of three above or below the median for their observing band are rejected. On average, 286 bolometers at $150 \mathrm{GHz}$ (out of 394 possible) passed all cuts for a given observation. At $220 \mathrm{GHz}$, an average of 161 (out of 254 possible bolometers) passed all cuts.

In a given observation, segments of the TOD corresponding to individual scans may be rejected due to readout or cosmic-ray induced features, anomalous noise, or problems with pointing reconstruction. The scan-byscan data cuts are the same as those described in S09 and remove approximately $10 \%$ of the TOD.

The SPT detectors exhibit some sensitivity to the receiver's pulse-tube cooler. Bolometer noise power spectra are occasionally contaminated by narrow spectral lines corresponding to the pulse-tube frequency and its harmonics. As in S09, we address this by applying a notch filter to the affected data. This filter removes less than $0.4 \%$ of the total signal bandwidth.

In addition to the cuts above, we remove a small number of observations that have either incomplete coverage of the field or high noise levels. Observations taken under poor atmospheric conditions are rejected in this way. The application of these cuts remove 36 out of the 336 total observations of the field.

\subsubsection{Time-Ordered Data (TOD) Filtering}

Let $d_{\alpha j}$ be a measurement of the CMB brightness temperature by detector $j$ at time $\alpha$. Contributions to $d_{\alpha j}$ are the celestial sky temperature, $s_{\alpha j}$, the atmospheric temperature, $a_{\alpha j}$, and instrumental noise, $n_{\alpha j}$. The instrumental noise is largely uncorrelated between detectors. However, the atmospheric signal is highly correlated across the focal plane due to the overlap of individual detector beams as they pass through turbulent layers in the atmosphere.

The signals in the data have been low-pass filtered by the bolometers' optical time constants. These single-pole bolometer transfer functions are measured by the method described in S09. We deconvolve the transfer functions for each bolometer on a by-scan basis, as the first step in TOD filtering. At the same time, we apply a lowpass filter with a cutoff at $12.5 \mathrm{~Hz}$ to remove noise above the Nyquist frequency associated with the final map pixelation. These operations can be represented as linear operation $\boldsymbol{\Pi}^{\mathrm{fft}}$ on the TOD,

$$
d_{\alpha j}^{\prime}=\Pi_{\alpha \beta}^{\mathrm{fft}} d_{\beta j},
$$

where a sum is implied over repeated indices.

To remove $1 / \mathrm{f}$ noise and atmospheric noise in the scan direction, we project out a 19th order Legendre polynomial from the TOD for each detector on a scan-by-scan basis. This operation $\boldsymbol{\Pi}^{\mathbf{t}}$ can be described by

$$
d_{\alpha j}^{\prime \prime}=\Pi_{\alpha \beta}^{\mathrm{t}} d_{\beta j}^{\prime}
$$

The effect on the signal is similar to having applied a one-dimensional high-pass filter in the scan direction at $\ell \gtrsim 300$.
Significant atmospheric signal remains in the data after this temporal polynomial removal. Because the atmospheric power is primarily common across the entire focal plane, we can exploit the spatial correlations to remove atmosphere without removing fine-scale astronomical signal. The method used in this analysis is to fit for and subtract a plane, $a_{0}+a_{x} \mathbf{x}+a_{y} \mathbf{y}$, across all detectors in the detector array at each time sample, where $\mathbf{x}$ and $\mathbf{y}$ are set by the angular separation of each pixel from the boresight of the telescope. All detectors are equally weighted in this fit. This operation can be represented as a linear operation on all detectors at each time sample $\alpha$ to produce an atmosphere-cleaned dataset, $\mathbf{d}^{\prime \prime \prime}$,

$$
d_{\alpha j}^{\prime \prime \prime}=\Pi_{j k}^{\mathrm{s}} d_{\alpha k}^{\prime \prime}
$$

We mask the brightest point sources in the map before applying the polynomial subtraction and the spatialmode filtering. The 92 sources that were detected above $5 \sigma$ in a preliminary $150 \mathrm{GHz}$ map have been masked. A complete discussion of the point sources detected in this field can be found in Vieira et al. (2009, hereafter V09).

\subsubsection{Map-making}

The data from each bolometer is inverse noise weighted according to the calibrated, pre-filtering detector PSD in the range $1-3 \mathrm{~Hz}$, corresponding to $1400<\ell<4300$. This multipole range covers the angular scales where we expect the most significant detection of the SZ effect. We represent the mapping between time-ordered bolometer samples and celestial positions with the pointing matrix $\mathbf{L}$, which we apply to the cleaned TOD to produce a map m,

$$
\mathbf{m}=\mathbf{L} \boldsymbol{\Lambda}_{\mathbf{w}} \boldsymbol{\Pi}^{\mathrm{s}} \boldsymbol{\Pi}^{\mathrm{t}} \boldsymbol{\Pi}^{\mathrm{fft}} \mathbf{d} .
$$

$\boldsymbol{\Lambda}_{\mathbf{w}}$ is a diagonal matrix encapsulating the detector weights. Information on the pointing reconstruction can be found in S09 and Carlstrom et al. (2009).

\subsection{Maps to Bandpowers}

We use a pseudo- $C_{\ell}$ method to estimate the bandpowers. In pseudo- $C_{\ell}$ methods, bandpowers are estimated directly from the Fourier transform of the map after correcting for effects such as TOD filtering, beams, and finite sky coverage. We process the data using a cross spectrum based analysis (Polenta et al. 2005; Tristram et al. 2005 in order to eliminate noise bias. Beam and filtering effects are corrected for according to the formalism in the MASTER algorithm (Hivon et al. 2002). We report the bandpowers in terms of $\mathcal{D}_{\ell}$, where

$$
\mathcal{D}_{\ell}=\frac{\ell(\ell+1)}{2 \pi} \mathrm{C}_{\ell} \text {. }
$$

As the first step in our analysis, we calculate the Fourier transform, $\tilde{m}_{\mathbf{k}}$, of the single-observation maps for each frequency. Each map is apodized using the same window $\mathbf{W}$, and thus $\tilde{m}_{\mathbf{k}}^{(\nu, A)} \equiv \mathrm{FT}\left(\mathbf{W} \mathbf{m}^{(\nu, A)}\right)$, where the first superscript, $\nu$, indicates the observing frequency, the second superscript, $A$, indicates the observation number, and the subscript denotes the angular frequency. Crossspectra are computed for every map pair, and averaged within the appropriate $\ell$-bin $b$ :

$$
D_{b}^{\nu_{i} \times \nu_{j}, A B} \equiv \frac{1}{N_{b}} \sum_{\mathbf{k} \in b} \frac{\mathrm{k}(\mathrm{k}+1)}{2 \pi} \tilde{m}_{\mathbf{k}}^{\left(\nu_{i}, A\right)} \tilde{m}_{\mathbf{k}}^{\left(\nu_{j}, B\right) *}
$$


We use the abbreviation, $D^{\nu_{i}} \equiv D^{\nu_{i} \times \nu_{i}}$, when referring to single-frequency auto-spectra. Recall that $|\mathbf{k}|=\ell$ in the flat-sky approximation.

The FFT of a map, $\widetilde{m}_{\mathbf{k}}^{(\nu, A)}$, is linearly related to the FFT of the astronomical sky, $a_{\mathbf{k}}^{\nu}$. It also includes a noise contribution, $n_{\mathbf{k}}^{(\nu, A i)}$, which is uncorrelated between the 300 observations:

$$
\widetilde{m}_{\mathbf{k}}^{(\nu, A)}=\widetilde{W}_{\mathbf{k}-\mathbf{k}^{\prime}} G_{\mathbf{k}^{\prime}}\left(B_{\mathbf{k}^{\prime}} a_{\mathbf{k}^{\prime}}^{\nu}+n_{\mathbf{k}^{\prime}}^{(\nu, A)}\right) .
$$

Here $G_{\mathbf{k}}$ is the 2-dimensional amplitude transfer function, which accounts for the TOD filtering as well as the map-based filtering described in $3.2 .2, \widetilde{\mathbf{W}}_{\mathbf{k}}$ is the Fourier transform of the apodization mask.

Following the treatment by Hivon et al. (2002), we take the raw spectrum, $D_{b}^{A B}$, to be linearly related to the true spectrum by a transformation, $K_{b b^{\prime}}$. The $K$ transformation combines the power spectrum transfer function, $F_{\ell}$, which includes the effects of TOD-based and map-based filtering ( 3.2 .3 , the beams, $B_{\ell}(2.1)$, and the modecoupling matrix, $M_{k k^{\prime}}[\mathbf{W}]$ (3.2.1). The mode-coupling matrix accounts for the convolution of the spectrum due to the apodization window, $\mathbf{W}$.

$$
K_{b b^{\prime}}^{\nu_{i} \times \nu_{j}}=P_{b k}\left(M_{k k^{\prime}}[\mathbf{W}] F_{k^{\prime}}^{\nu_{i} \times \nu_{j}} B_{k^{\prime}}^{\nu_{i}} B_{k^{\prime}}^{\nu_{j}}\right) Q_{k^{\prime} b^{\prime}} .
$$

Here, $P_{b k}$ is the re-binning operator (Hivon et al. 2002):

$$
P_{b k}=\left\{\begin{array}{cl}
\frac{k(k+1)}{2 \pi \Delta k_{(b)}} & k_{(b-1)}<k<k_{(b)} \\
0 & \text { otherwise }
\end{array}\right.
$$

while the inverse of the re-binning operator is $Q_{k b}$ :

$$
Q_{k b}=\left\{\begin{array}{cl}
\frac{2 \pi}{k(k+1)} & k_{(b-1)}<k<k_{(b)} \\
0 & \text { otherwise }
\end{array} .\right.
$$

In the rest of the paper, we will often refer to band averaged quantities, such as $C_{b}=P_{b k} C_{k}$. Cross-spectra between observations that have been corrected for apodization and processing are denoted as,

$$
\widehat{D}_{b}^{\nu_{i} \times \nu_{j}, A B} \equiv\left(K^{-1}\right)_{b b^{\prime}} D_{b^{\prime}}^{\nu_{i} \times \nu_{j}, A B} .
$$

The final bandpowers are then computed as the average of all cross-spectra,

$$
q_{b}^{\nu_{i} \times \nu_{j}}=\frac{1}{n_{\mathrm{obs}}\left(n_{\mathrm{obs}}-1\right)} \sum_{A} \sum_{B \neq A} \widehat{D}_{b}^{\nu_{i} \times \nu_{j}, A B} .
$$

We make the simplifying assumption that the noise properties of each observation are statistically equivalent, hence the uniform weighting chosen here. The data selection criteria in 3.1.1 ensure that these observations target the same region of the sky, have roughly the same number of active bolometers, and have similar noise properties. Therefore, this uniform weighting should be unbiased and only slightly sub-optimal.

The cross-spectrum bandpowers, $D_{b}^{\nu_{i} \times \nu_{j}, A B}$, generated from the 300 observations are used in conjunction with signal-only Monte Carlo bandpowers to generate empirical covariance matrices, as described in appendix A. The variance of the Monte Carlo bandpowers is used to estimate the sample variance contribution to the covariance matrix. Meanwhile, we use the variance in the spectra of the real data to estimate the uncertainty due to noise in the maps.

\subsubsection{Apodization Mask and Calculation of the Mode-mixing Kernel}

Since we have only mapped a fraction of the full sky, the angular power spectra of the maps are convolutions of the true $C_{\ell} \mathrm{s}$ with an $\ell$-space, mode-mixing kernel that depends on the map size, apodization, and point source masking. We calculate this mode-mixing kernel, $M_{k k^{\prime}}[\mathbf{W}]$, following the derivation in Hivon et al. (2002) for the flat sky case:

$$
M_{k k^{\prime}}[\mathbf{W}] \equiv \frac{1}{(2 \pi)^{2}} \int d \theta_{k} d \theta_{k^{\prime}}\left|\widetilde{W}_{\mathbf{k}-\mathbf{k}^{\prime}}\right|^{2} .
$$

If the mask is smooth on fine angular scales, then the mode coupling kernel can be approximated by a delta function at high- $k$ :

$$
M_{k k^{\prime}}[\mathbf{W}] \approx w_{2} \delta_{k k^{\prime}} .
$$

Here we use the notation $w_{n} \equiv\left\langle\mathbf{W}^{n}\right\rangle$ to represent the $n^{\text {th }}$ moment of the apodization window. In this limit, the coupling kernel serves the purpose of re-normalizing the power spectrum to account for modes lost due to apodization. As we discuss in appendix A, the coupling kernel also plays an important role in determining the shape and normalization of the covariance matrix.

We avoid areas of the map with sparse or uneven coverage in any single observation. Thus, the apodization window is conservative in its avoidance of the map edges. We also mask 144 point sources detected in the $150 \mathrm{GHz}$ data above a significance of $5 \sigma$, which corresponds to a flux of $6.4 \mathrm{mJy}$. This source list is from a more refined analysis than the preliminary list used in $\$ 3.1 .2$, the differences are in sources near the $5 \sigma$ detection threshold. Each point source is masked by a 2'-radius disk, with a Gaussian taper outside this radius. Many different tapered mask shapes were tested for both efficacy in removing point source power and noise performance. Given the relatively small area masked by point sources, varying the shape of the point source mask has little effect on the final spectrum. The effective sky area of the mask is78 $\mathrm{deg}^{2}$. Simulations have been performed to test whether the application of this mask will bias the inferred power and we see no bias with a $5 \sigma$ cut. As could be expected, we do observe a mild noise bias when using a more aggressive $3 \sigma$ point source mask. The same $5 \sigma$ mask is used for all maps at both frequencies and for all observations.

\subsubsection{Fourier Mode Weighting}

The maps produced by the steps described in $\$ 3.1 .2$ have anisotropic signal and noise. In particular, the map noise PSD rises steeply at spatial frequencies corresponding to low spatial frequencies in the scan direction (low $k_{x}$ ). The covariance of the power estimated in a given $\ell$-bin depends on the second power of the map noise PSD for all the Fourier modes in that $\ell$-bin. In the presence of either non-uniform noise or signal, applying an optimal mode-weighting when calculating the mean bandpower may significantly reduce the final noise covariance matrix of the power spectrum bandpowers. In the case of SPT, we find that for the purposes of measuring the $\ell \gtrsim 2000$ 
power spectrum, a simple, uniform selection of modes at $k_{x}>1200$ is close to optimal, and we apply this mode weighting in calculating the SPT bandpowers.

\subsubsection{Transfer Function Estimation}

In order to empirically determine the effect of both the TOD-based filtering and the Fourier mode-weighting, we calculate a transfer function, $F_{k}$, as defined in Hivon et al. (2002). Note that this power spectrum transfer function is distinct from the amplitude transfer function $G_{\mathbf{k}}$. Specifically, the transfer function accounts for all map- and TOD-processing not taken into account by the mode-coupling kernel or the beam functions.

We created 300 Monte Carlo sky realizations at 150 and $220 \mathrm{GHz}$ in order to calculate the transfer function of the filtering. The simulations also serve as an input for the covariance matrix estimation. These simulations contain two components: a CMB component and a point source component. The CMB component is computed for the best-fit WMAP5 lensed $\Lambda$ CDM model.

The point source component includes two different populations of dusty galaxies, a low-z population and a high-z population. For each population we generate sources from a Poisson distribution. Sources are generated in bins of flux, $S$, ranging from 0.01 to $6.75 \mathrm{mJy}$. This upper limit in flux is close to the $5 \sigma$ detection threshold in the $150 \mathrm{GHz}$ maps. In each flux bin, the $150 \mathrm{GHz}$ source density, $d N / d S$, of each population is taken from the model of Negrello et al. (2007). We relate the flux of each source at $220 \mathrm{GHz}$ to its Hux at $150 \mathrm{GHz}$ with a power law in intensity, $S_{\nu} \propto \nu^{\alpha}$. The power law spectral index, $\alpha$, for each source is drawn from a normal distribution. We use spectral indices of $\alpha=3 \pm 0.5$ for the high-z protospheroidal galaxies, and $\alpha=2 \pm 0.3$ for the low-z IRAS-like galaxies. As with any Poisson distribution of point sources, the power spectrum of these maps is white $\left(C_{\ell}^{\mathrm{ps}}=\right.$ constant $)$ and related to the flux cutoff, $S_{0}$, by:

$$
C_{\ell}^{\mathrm{ps}}=\left(\frac{d B_{\nu}}{d T}\right)_{\mathrm{T}_{\mathrm{CMB}}}^{-2} \int_{0}^{S_{0}} S^{2} \frac{d N}{d S} d S
$$

The power spectra of these simulated point source maps are well represented by a constant $C_{\ell}^{\mathrm{ps}}=1.1 \times 10^{-5} \mu \mathrm{K}^{2}$ at $150 \mathrm{GHz}$ and $C_{\ell}^{\mathrm{ps}}=6.8 \times 10^{-5} \mu \mathrm{K}^{2}$ at $220 \mathrm{GHz}$.

These simulated maps are smoothed by the appropriate beam. From each map realization, we construct simulated TOD using the pointing information. Each realization of the TOD is processed using the low-pass filter, polynomial removal, and the spatial-mode subtraction described in 3.1 .2 . No time-constant deconvolution is applied, since these realizations of the TOD have not been convolved by the bolometer time constants. The filtered, simulated TOD are then converted into maps according to equation 7

We apply the same apodization mask and Fourier mode weighting to these map realizations as is used on the actual data. We then compute the Monte Carlo pseudopower spectrum, $\left(D_{k}\right)_{\mathrm{MC}}$, for each map. The simulated transfer function is calculated iteratively, by comparing the Monte Carlo average, $\left\langle D_{k}\right\rangle_{\mathrm{MC}}$, to the input theory spectrum, $C_{k}^{\text {theory }}$.

For the single-frequency bandpowers, we start with an initial guess of the transfer function:

$$
F_{k}^{\nu,(0)}=\frac{\left\langle D_{k}^{\nu}\right\rangle_{\mathrm{MC}}}{w_{2} B_{k}^{\nu} C_{k}^{\nu, \text { theory }}} .
$$

The superscript, $(0)$, indicates that this is the first iteration in the transfer function estimates. For this initial guess, we approximate the coupling kernel as largely diagonal as in equation 17. Thus, the factor $w_{2}$ is the normalization factor required by the apodization window. We then iterate on this estimate using the full modecoupling kernel:

$$
F_{k}^{\nu,(i+1)}=F_{k}^{\nu,(i)}+\frac{\left\langle D_{k}^{\nu}\right\rangle_{\mathrm{MC}}-M_{k k^{\prime}} F_{k^{\prime}}^{\nu,(i)} B_{k^{\prime}}^{\nu} C_{k^{\prime}}^{\nu, \text { theory }}}{B_{k}^{\nu 2} C_{k}^{\nu, \text { theory }} w_{2}} .
$$

We iterate on this estimate five times, although the transfer function has largely converged after the first iteration.

This method may misestimate the transfer function if the simulated spectrum is significantly different from the true power spectrum. The primary CMB anisotropy has been adequately constrained by previous experiments, however, the foreground power spectrum is less well known. We repeated the transfer function estimation using an input power spectrum with twice the nominal point source power. The resulting transfer function was unchanged at the $1 \%$ level, giving confidence that the transfer function estimate is robust.

The transfer function for a multifrequency crossspectrum is taken to be the geometric mean of the two individual transfer functions:

$$
F_{k}^{\nu_{i} \times \nu_{j}}=\sqrt{F_{k}^{\nu_{i}} F_{k}^{\nu_{j}}}
$$

This treatment is only strictly correct for isotropic filtering. As a cross check, we have also computed the cross-spectrum transfer function directly. For the angular multipoles reported in this work, the geometric-mean transfer function estimate is in excellent agreement with the estimate obtained using $\left\langle D_{k}^{\nu_{1} \times \nu_{2}}\right\rangle_{\mathrm{MC}}$ in equations 19 and 20 .

\subsubsection{Frequency-differenced Spectra}

We are interested in the power spectra of linear combinations of the 150 and $220 \mathrm{GHz}$ maps designed to remove astronomical foregrounds. One method for obtaining such power spectra would be to directly subtract the maps after correcting the maps for differences in beams or processing. In such a map subtraction, the scaling of the $220 \mathrm{GHz}$ map, $x$, can be adjusted to optimally remove foregrounds. The differenced maps can then be processed using our standard pipeline to obtain spectra with a reduced foreground contribution.

Equivalently, the differenced spectrum can be generated from the original bandpowers, $q_{b}^{i}$, using a linear spectrum transformation, $\xi$ :

$$
q_{b}^{150-x \times 220}=\sum_{i} \xi^{i}(x) q_{b}^{i}
$$

Here, the index, $i$, denotes the $150 \mathrm{GHz}$ auto-spectrum, $220 \mathrm{GHz}$ auto-spectrum, or $150 \times 220 \mathrm{GHz}$ cross spectrum. This transformation is computationally fast and takes advantage of the fact that the cross-frequency 
bandpowers include information on the relative phases of each Fourier component in the map. In this way, the difference spectrum can be represented in terms of the three measured spectra:

$$
\begin{aligned}
q_{b}^{\nu_{i}-x \times \nu_{j}} & =\frac{1}{(1-x)^{2}} \sum_{\mathbf{k} \in b}\left|a_{\mathbf{k}}^{\nu_{i}}-x a_{\mathbf{k}}^{\nu_{j}}\right|^{2} \\
& =\frac{1}{(1-x)^{2}} \sum_{\mathbf{k} \in b}\left|a_{\mathbf{k}}^{\nu_{i}}\right|^{2}-2 x \operatorname{Re}\left(a_{\mathbf{k}}^{\nu_{i}} a_{\mathbf{k}}^{\nu_{j} *}\right)+x^{2}\left|a_{\mathbf{k}}^{\nu_{j}}\right|^{2} \\
& =\frac{1}{(1-x)^{2}}\left(q_{b}^{\nu_{i}}-2 x q_{b}^{\nu_{i} \times \nu_{j}}+x^{2} q_{b}^{\nu_{j}}\right)
\end{aligned}
$$

The overall normalization is chosen such that the CMB power is unchanged in the subtracted spectrum. For clarity, we have momentarily avoided here the complications of beams and filtering and have expressed the bandpowers in terms of the Fourier transform of the celestial sky, $a_{\mathbf{k}}$. For a given proportionality constant, $x$, the values of $\xi(x)$ are:

$$
\begin{aligned}
\xi^{150}(x) & =\frac{1}{(1-x)^{2}}, \\
\xi^{150 \times 220}(x) & =\frac{-2 x}{(1-x)^{2}}, \\
\xi^{220}(x) & =\frac{x^{2}}{(1-x)^{2}} .
\end{aligned}
$$

We also compute the covariance matrix for the subtracted spectrum from the original bandpower covariance matrices:

$$
\mathbf{C}_{b b^{\prime}}^{150-x \times 220}=\sum_{i, j} \xi^{i}(x) \mathbf{C}_{b b^{\prime}}^{(i, j)} \xi^{j}(x) .
$$

\section{JACK-KNIVES}

We apply a set of jack-knife tests to the SPT data to search for possible systematic errors. In a jack-knife test, the data set is divided into two halves, based on features of the data associated with potential sources of systematic error. The two halves are differenced to remove any astronomical signal, and the resulting power spectrum is compared to zero. Significant deviations from zero would indicate either a systematic problem or a noise misestimate. We implement the jack-knives in the crossspectrum framework by differencing single pairs of observations and applying the cross-spectrum estimator outlined in $\$ 3.2$ to the set of differenced pairs. In total, we perform 13 different jack-knife tests.

Six jack-knives are based on the observing parameters, such as time, scan direction and azimuthal range. The data can be split based on when it was taken to search for systematic changes in the calibration, beams, detector time constants, or any other time variable effect. The "first half - second half" jack-knife probes variations on month time scales, while an "even-odd" jack-knife differencing every other observation looks for variations on hourly time scales. Results for the "first half - second half" jack-knife are shown in the top panel of Figure 2 The data can also be split based on the direction of the scan in a "left-right" jack-knife (panel 2 of Figure 2). We would expect to see residual power here if the detector transfer function had been improperly de-convolved, if the telescope acceleration at turn-arounds induces a signal through sky modulation or microphonics, or if the wind direction is important. We observed this field at two scan speeds different by $10 \%$. We check for systematic differences related to the scan speed, such as a mirror wobble, in a "scan speed" jack-knife. Side-lobe pickup could potentially introduce spurious signals into the SPT maps from the moon or features on the ground. We test for moon pickup by splitting the data based on whether the moon is above or below the horizon. We test for ground pickup by splitting on the mean azimuth of the observation. To maximize the sensitivity to ground pickup, the azimuthal ranges are selected to be centered on and directly opposite the closest building to the telescope, which is the most likely source of ground signal. The azimuthal jack-knife is shown in the third panel of Figure 2,

We also perform jack-knives based on four noise and observation-quality statistics of the $150 \mathrm{GHz}$ data. The first is based on the overall RMS in the maps, which is affected by atmospheric conditions and detector noise. The second is based on the average raw detector PSD in the range $9-11 \mathrm{~Hz}$, which is a measure of the detector "white" noise level. The third is the RMS near $\ell=3000$ where the $\mathrm{S} / \mathrm{N}$ on the $\mathrm{SZ}$ power spectrum is highest. The fourth is based on the number of bolometers active in each observation.

There are also a number of line-like spectral features in the SPT TOD that could potentially affect the power spectrum bandpowers, and we perform three jacknife tests for sensitivity to these features. Some of these line features appear at harmonics of the receiver pulsetube frequency, and are typically correlated across many bolometer channels. These lines are filtered from the data as described in 33.1 .2 . In addition, some channels exhibit occasional line-like features at other frequencies, which are not filtered in the data processing. We search for residual effects in a jack-knife based on the average number of line-like features for all $150 \mathrm{GHz}$ bolometers, as well as a jack-knife based on the bandwidth affected by the lines. Finally, we do an additional split using the average number of lines in an observation that appear to be related to the pulse-tube cooler.

We calculate the $\chi^{2}$ with respect to zero for each jack-knife over the range $\ell \in[2000,10000]$ in bins with $\Delta \ell=500$. Some of the tests are highly correlated. For example, we changed scan velocities approximately midway through the observations so splitting the data based on scan velocity is nearly identical to splitting the data between the first and second halves of the season. We calculate a correlation coefficient between the different tests by adding $1 / N_{o b s}$ for each common observation in a half, and subtracting $1 / N_{o b s}$ for each distinct observation in a half. This algorithm returns unity for two identical sets and zero for two random sets, as we expect $50 \%$ of the observations to be in common for two random selections. The correlation coefficients between the 13 jack-knives range from 0 to 0.83 , with the maximum correlation being for the previously mentioned scan velocity and first half - second half jack-knives. We invert the jack-knife correlation matrix, $\mathcal{C}$, and calculate $\chi^{2}=v_{i \alpha}\left(\mathcal{C}^{-1}\right)_{i j} v_{j \alpha}$. Here $v_{i \alpha}$ is the ratio of the bandpower over the uncertainty for the $i^{\text {th }}$ jack-knife and $\alpha^{\text {th }}$ 
$\ell$-bin. The probability to exceed the measured $\chi^{2}$ for the complete set of thirteen jack-knives is $77 \%$ for the $150 \mathrm{GHz}$ data, $32 \%$ for the $220 \mathrm{GHz}$ data and $57 \%$ for the combined set with both frequencies. We thus find no evidence for systematic contaminants in the SPT data set.

\section{FOREGROUNDS}

The main foregrounds at frequencies near $150 \mathrm{GHz}$ are expected to be galactic dust emission, radio sources, and dusty star forming galaxies (DSFGs). Note, however, that the SPT field is selected to target one of the cleanest regions on the sky for galactic dust emission, and in the Finkbeiner et al. (1999) model, dust emission is primarily on large angular scales. The contribution for the selected field on arcminute scales is insignificant. The primary foregrounds of consideration for this analysis are radio sources and DSFGs.

Tens of bright radio sources are detected in the SPT maps at $>5 \sigma$, and contribute substantial amounts of power at both 150 and $220 \mathrm{GHz}$. Information on the fluxes and spectral indices of these and other sources significantly detected in the SPT maps can be found in V09. Without masking, the measured point source power is $C_{\ell}^{\text {unmasked }}=2.1 \times 10^{-4} \mu \mathrm{K}^{2}$ at $150 \mathrm{GHz}$ and $C_{\ell}^{\text {unmasked }}=1.6 \times 10^{-4} \mu \mathrm{K}^{2}$ at $220 \mathrm{GHz}$. These estimates are dominated by the brightest few sources and thus subject to very large sample variance. We mask all sources with $150 \mathrm{GHz}$ fluxes above the $5 \sigma$ detection threshold, $S=6.4 \mathrm{mJy}$. By masking these sources we reduce the radio source contribution to the SPT bandpowers by several orders of magnitude. According to the de Zotti et al. (2005) model source counts, after masking bright sources, we expect a residual radio source contribution of $C_{\ell}^{\text {radio }}=8.5 \times 10^{-7} \mu \mathrm{K}^{2}$ at $150 \mathrm{GHz}$.

The point source masking will remove the SZ contribution from only a few galaxy clusters, leading to negligible reduction of the SZ power. This is because the large majority of radio sources and DSFGs reside outside of SZ clusters. Most of the masked sources are identified as radio sources in V09. Extrapolations from lower frequency observations imply that, at $150 \mathrm{GHz}$, less than $3 \%$ of clusters contain radio source flux exceeding $20 \%$ of the tSZ flux decrement (Lin et al. 2009; Sehgal et al. 2009). The masked sources are selected as increments at $150 \mathrm{GHz}$ and therefore have fluxes much greater than $20 \%$ of the tSZ of any associated galaxy cluster. The number of clusters masked by the radio source masking should then be much less than $3 \%$ and negligible. We also compare the tSZ power spectrum in the Sehgal et al. (2009) simulated sky maps with and without masking $>6.4 \mathrm{mJy}$ sources and find the difference to be $<<1 \%$. A small number (six) of the masked sources are identified as DSFGs (V09). Galaxy clusters have a DSFG abundance only twenty times larger than the field (Bai et al. 2007), although they exceed the mass density of the field by a factor of 200 or more. Given the relative rarity of galaxy clusters, it follows that only a small fraction of DSFGs can live in galaxy clusters. Therefore, the number of clusters masked along with the DSFGs should be much smaller than six and negligible.

Both the DSFG and radio source arguments above depend implicitly on the impact of potentially masking $\lesssim 10$ clusters. As a worst-case study, Shaw et al. (2009) consider the impact of masking the most massive ten clusters in the field and show that even in this extreme case, the tSZ power spectrum at $\ell=3000$ is reduced by only $11 \%$. Of course, the point source masking will not select the most massive clusters and is highly unlikely to remove as many as ten clusters. Hence, the true impact will be significantly less.

After we mask sources above the $5 \sigma$ threshold, DSFGs are the dominant point source population in the SPT maps. These sources have been extensively studied at higher frequencies by SCUBA (Holland et al. 1999), MAMBO (Kreysa et al. 1998), Bolocam (Glenn et al. 1998), LABOCA (Siringo et al. 2009), AzTEC (Scott et al. 2008), SCUBA-2 (Holland et al. 2006), and BLAST (Pascale et al. 2008), and there have been some preliminary indications of their contribution in previous small-scale power spectra at $150 \mathrm{GHz}$ (Reichardt et al. $2009 \mathrm{a}$ b). The flux of these galaxies has been observed to scale to higher frequencies as $S_{\nu} \propto \nu^{2.4-3.0}$ (Knox et al. 2004. Greve et al. 2004, Reichardt et al. 2009b), with the exact frequency dependence a function of the dust emissivity, the dust temperature, and the redshift distribution of the galaxy population. This range of spectral indices corresponds to point source amplitude ratios, $\delta T_{220}^{\mathrm{ps}} / \delta T_{150}^{\mathrm{ps}}$, of 2.1-2.6 when expressed in units of CMB temperature. The measured spectral index of the DSFGs in the SPT maps is discussed extensively in H09.

In order to obtain an unbiased estimate of the SZ power spectrum, it is essential that we take these sources into account in our fits and modeling. After masking the bright point sources, we significantly detect a Poisson distributed power at $150 \mathrm{GHz}$ of $C_{\ell}^{\mathrm{ps}}=7.1 \pm 0.5 \times 10^{-6} \mu \mathrm{K}^{2}$ (H09). This unclustered point source power climbs with increasing $\ell$ to be comparable to the SZ effect by $\ell=$ $2500-3000$, and is the dominant astronomical signal in the maps at arcminute scales.

The distribution of DSFGs on the sky is also expected to be clustered, resulting in a significant increase in power at $\ell \lesssim 3000$. BLAST recently detected this clustered term for DSFGs at $600-1200 \mathrm{GHz}(500-250 \mu \mathrm{m})$ (Viero et al. 2009). Extrapolating the measured clustering to $150 \mathrm{GHz}$, we expect the clustered contribution to be comparable to the tSZ effect. H09 have analyzed the SPT 150, 220, and $150 \times 220 \mathrm{GHz}$ bandpowers presented in 86 and have also found that the amplitude of the clustered component is indeed comparable to the tSZ amplitude. Discriminating between clustered DSFGs and the tSZ effect would be extremely difficult for a singlefrequency instrument as the angular dependencies are very similar. However, the two frequencies used in this analysis allow the spectral separation of the these two astronomical signals.

\section{RESULTS}

In addition to presenting the SPT angular power spectra, we wish to determine the amplitude of the tSZ power spectrum and to use this amplitude to constrain the normalization of the matter power spectrum, $\sigma_{8}$. This measurement requires separating the tSZ signal from the other astrophysical signals in our data which include primary CMB anisotropy (including lensing effects), DSFGs (both Poisson and clustered components), and anisotropy due to the kSZ effect. The primary CMB anisotropy and Poisson point source component can be 


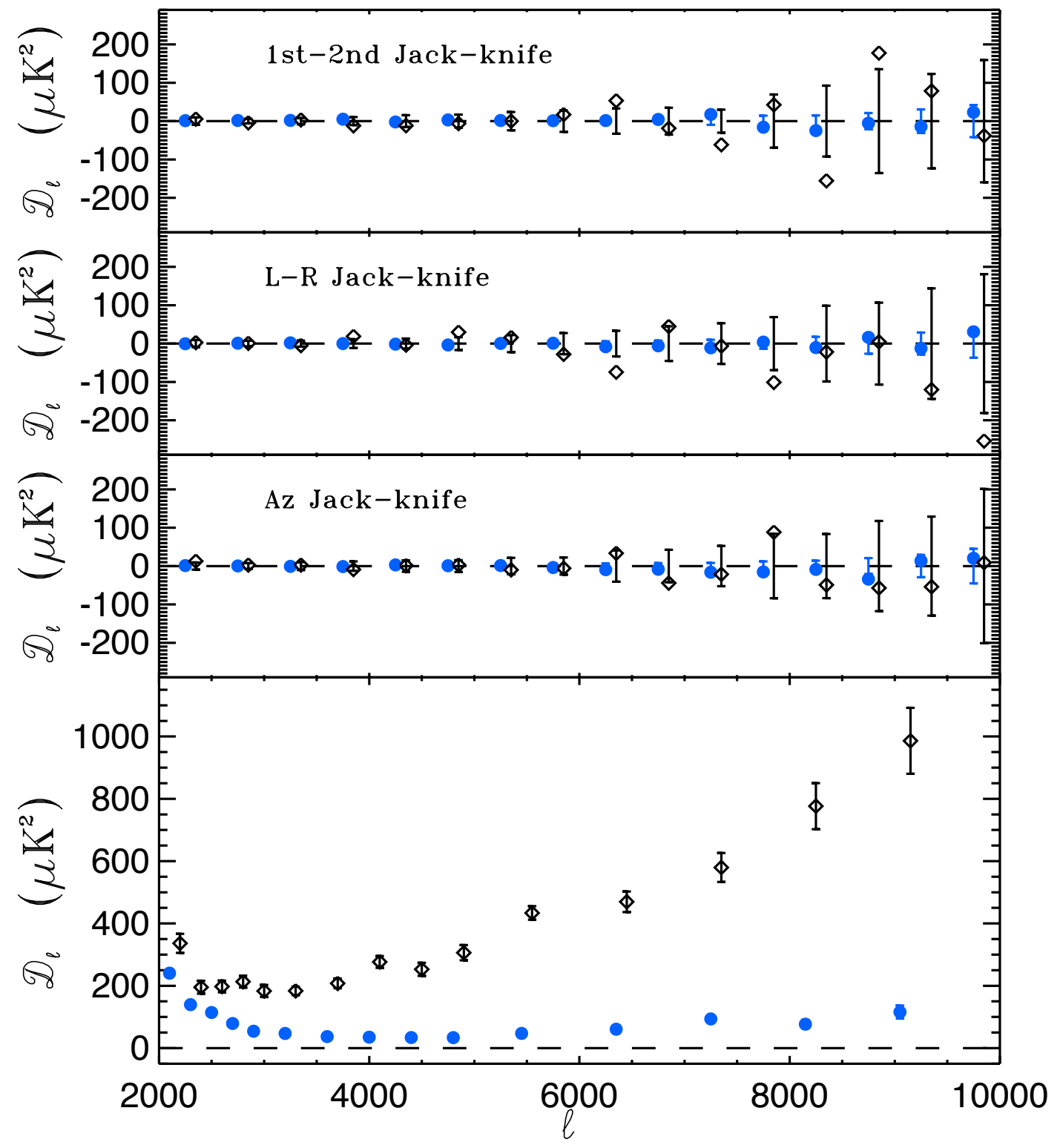

FIG. 2. - Jack-knives for the SPT data set at $150 \mathrm{GHz}$ (blue circles) and $220 \mathrm{GHz}$ (black diamonds). For clarity, the $220 \mathrm{GHz}$ jack-knives have been shifted to the right by $\Delta \ell=100$. Top panel: Bandpowers of the "first half - second half" jack-knife compared to the expected error bars about zero signal. Disagreement with zero would indicate either a noise misestimate or a time-dependent systematic signal. Second panel: Power spectrum of the left-going minus right-going difference map. This test yields strong constraints on the accuracy of the detector transfer function deconvolution and on possible directional systematics. Third panel: Bandpowers for the difference map when the data is split based on azimuth. Signals fixed in azimuth such as side-lobe pickup from the nearby support building would produce non-zero power. We see no evidence for ground-based pickup. The cumulative probability to exceed the $\chi^{2}$ observed in these three tests is $76 \%$ at $150 \mathrm{GHz}$ and $22 \%$ at $220 \mathrm{GHz}$. Bottom panel: The un-differenced SPT power spectra at each frequency for comparison.

separated from a tSZ-like component on account of the distinct angular power spectra of these three signals. However the tSZ, kSZ, and clustered DSFG components are all expected to be roughly flat in $\mathcal{D}_{\ell}$, and we depend on their distinct frequency dependences to separate them. We use a combination of the two frequency bands to remove the DSFG contribution to the power spectra. We address the remaining degeneracy between the tSZ and $\mathrm{kSZ}$ effects by repeating the analysis for a range of assumed kSZ models.

In $\$ 6.1$ we present bandpowers for 150,220 and $150 \times$ $220 \mathrm{GHz}$ SPT angular power spectra. In $\$ 6.2$ we combine the two frequency bands to generate a set of DSFGsubtracted bandpowers. In $\$ 6.3$ we describe the Monte
Carlo Markov chain (MCMC) analysis used to estimate the tSZ power spectrum amplitude, parametrized as the normalization $A_{\mathrm{SZ}}$ of a model template, from the DSFGsubtracted bandpowers. In 6.4 we discuss the implications of the measured tSZ power spectrum amplitude for $\sigma_{8}$ and modeling of the tSZ effect.

\subsection{Power Spectra}

Figure 3 shows the bandpowers we compute by applying the analysis methods described in $\$ 3$ to one $100 \mathrm{deg}^{2}$ field observed by SPT at 150 and $220 \mathrm{GHz}$. The bandpowers for the two frequencies and their cross-spectrum are tabulated in Table 1. We combine these multifrequency data as described in $\$ 3$ to produce the 'DSFG- 
subtracted' bandpowers listed in Table 2. This power spectrum is compared to the results from WMAP5, ACBAR, and QUaD in Figure 4 . The best-fit model to this combined data set including the primary CMB, $\mathrm{kSZ}$, tSZ, and a Poisson point source contribution is shown for reference. The primary $\mathrm{CMB}$ anisotropy is estimated for a spatially-flat, $\Lambda$ CDM model, which includes gravitational lensing. The bandpower uncertainties are derived from the combination of simulations and the measured intrinsic variations within the SPT data as described in 93. The bandpowers can be compared to theory using the associated window functions (Knox 1999). The bandpowers, uncertainties, and window functions may be downloaded from the SPT website. ${ }^{25}$

The SPT bandpowers ae dominated by the damping tail of the primary CMB anisotropy on angular multipoles $2000<\ell<3000$. At these multipoles, the bandpowers are in excellent agreement with the predictions of a $\Lambda \mathrm{CDM}$ model determined from CMB observations on larger angular scales. On smaller scales, the SPT bandpowers provide new information on secondary CMB anisotropies and foregrounds which dominate the primary CMB anisotropy. The SPT data presented here represent the first highly significant detection of power at these frequencies and angular scales where the primary CMB anisotropy is sub-dominant. After masking bright point sources, the total signal-to-noise ratios on power in excess of the primary CMB are 55, 55, and 45 at $150,150 \times 220$, and $220 \mathrm{GHz}$ respectively. The majority of the high- $\ell$ power can be attributed to a Poisson distribution of point sources (likely DSFGs) on the sky. H09 use the bandpowers presented in this work to measure both Poisson and clustered contributions to the measured powers and investigate the implications for the properties of DSFGs. The largest source of secondary CMB anisotropy at $150 \mathrm{GHz}$ is expected to be the SZ effect, and we investigate SZ constraints in the following sections.

\subsection{DSFG-subtracted Bandpowers}

Our immediate goal is to measure the amplitude of the tSZ power spectrum. However, several signals in these maps have similar angular power spectrum shapes, and the single-frequency maps only constrain the sum of the power from these sources. For instance, the $150 \mathrm{GHz}$ data effectively constrain the sum of the tSZ, kSZ, and clustered DSFG power.

As discussed earlier, each of these components has a distinct frequency dependence so a linear combination of SPT's two frequency bands can be constructed (following 3.2.4 to minimize any one of them. H09 find significant evidence for a clustered DSFG power contribution to the single-frequency bandpowers listed in Table 1 with an amplitude comparable to that of the tSZ effect. We expect the $\mathrm{kSZ}$ effect to be smaller than the tSZ effect at $150 \mathrm{GHz}$ on theoretical grounds. Additionally, due to the frequency dependencies of the components, removing the $\mathrm{kSZ}$ effect would inflate the relative contribution of clustered DSFGs with respect to the tSZ effect. Therefore, we choose to remove DSFGs from the SPT bandpowers.

For a mean DSFG spectral index, $\alpha$, the proper weighting ratio, $x$, to apply to the $220 \mathrm{GHz}$ spectrum for DSFG

25 http://pole.uchicago.edu/public/data/lueker09/ removal would be:

$$
x=\frac{S_{150} /\left(\left.\frac{d B_{\nu}}{d T_{\mathrm{CMB}}}\right|_{150}\right)}{S_{220} /\left(\left.\frac{d B_{\nu}}{d T_{\mathrm{CMB}}}\right|_{220}\right)}=(150 / 220)^{\alpha} \frac{\left.\frac{d B_{\nu}}{d T_{\mathrm{CMB}}}\right|_{220}}{\left.\frac{d B_{\nu}}{d T_{\mathrm{CMB}}}\right|_{150}} .
$$

The spectrum in Table 2 and Figures 4 and 5 is produced with a weighting factor of $x=0.325$ corresponding to a mean spectral index of $\alpha=3.6$. The contribution from DSFGs can be completely removed only if every galaxy has the same spectral index. However, the comparative closeness of SPT's two frequency bands ensures that power leakage into the difference maps remains small despite some expected scatter in the spectral index of the dusty galaxies. In 6.2 .1 , we motivate this choice of $x$ and discuss predictions for the residual point source power.

We assume that there is no tSZ contribution to the $220 \mathrm{GHz}$ data as the $220 \mathrm{GHz}$ band is designed to be centered on the SZ null. Fourier transform spectroscopy measurements of the $220 \mathrm{GHz}$ band pass confirm that the tSZ amplitude in the $220 \mathrm{GHz}$ band will be $\lesssim 5 \%$ of the $150 \mathrm{GHz}$ amplitude. Any error incurred by subtracting roughly one third of the $220 \mathrm{GHz}$ amplitude from the $150 \mathrm{GHz}$ data would be less than $3 \%$, far below the present $\sim 40 \%$ statistical uncertainty on $A_{\mathrm{SZ}}$ (see Table 3).

It is important to note that the apparent tSZ power in the DSFG-subtracted bandpowers will be a factor of $1 /(1-x)^{2}=2.2$ higher than at $150 \mathrm{GHz}$ as the differenced bandpowers have been normalized to preserve the amplitude of the primary CMB anisotropy. In this work, we report SZ amplitudes scaled to $153 \mathrm{GHz}$ which is the effective band center of the $150 \mathrm{GHz}$ band for a tSZ spectrum.

\subsubsection{Residual Point Source Power}

The DSFG-subtracted maps have substantially less power due to both unclustered and clustered point sources as seen in Figure 5. However, we include a Poisson point source amplitude in all fits since we expect a small fraction of the point source power to remain in the DSFG-subtracted maps. The best-fit amplitude is consistent with zero and unphysical negative values of the Poisson point source power are allowed due to noise. To prevent this, we place a prior on the residual Poisson point source power based on what we know about the observed DSFG population from H09 and radio source population from V09 and de Zotti et al. (2005).

The residual power in the subtracted map due to Poisson-distributed DSFGs, $C_{\ell}^{\mathrm{DSFG}}$, depends on the scatter in spectral indices $\sigma_{\alpha}$, the accuracy to which the mean spectral index $\bar{\alpha}$ is known, and an estimate of the Poisson DSFG power in the $150 \mathrm{GHz}$ band, $C_{\ell}^{\mathrm{ps}, 150}$. For a given combination of these parameters, this residual DSFG power will be:

$$
\begin{aligned}
C_{\ell}^{\mathrm{DSFG}}= & C_{\ell}^{\mathrm{ps}, 150} \times \\
& \left(\sigma_{\alpha}^{2}\left[\ln \left(\nu_{150} / \nu_{220}\right)\right]^{2}+\left(1-\frac{x}{x_{\text {true }}}\right)^{2}\right),
\end{aligned}
$$

where $\nu_{150}$ and $\nu_{220}$ are the effective bandcenters of the 150 and $220 \mathrm{GHz}$ bands, and $x$ and $x_{\text {true }}$ are the assumed and true values of map weighting ratio. 


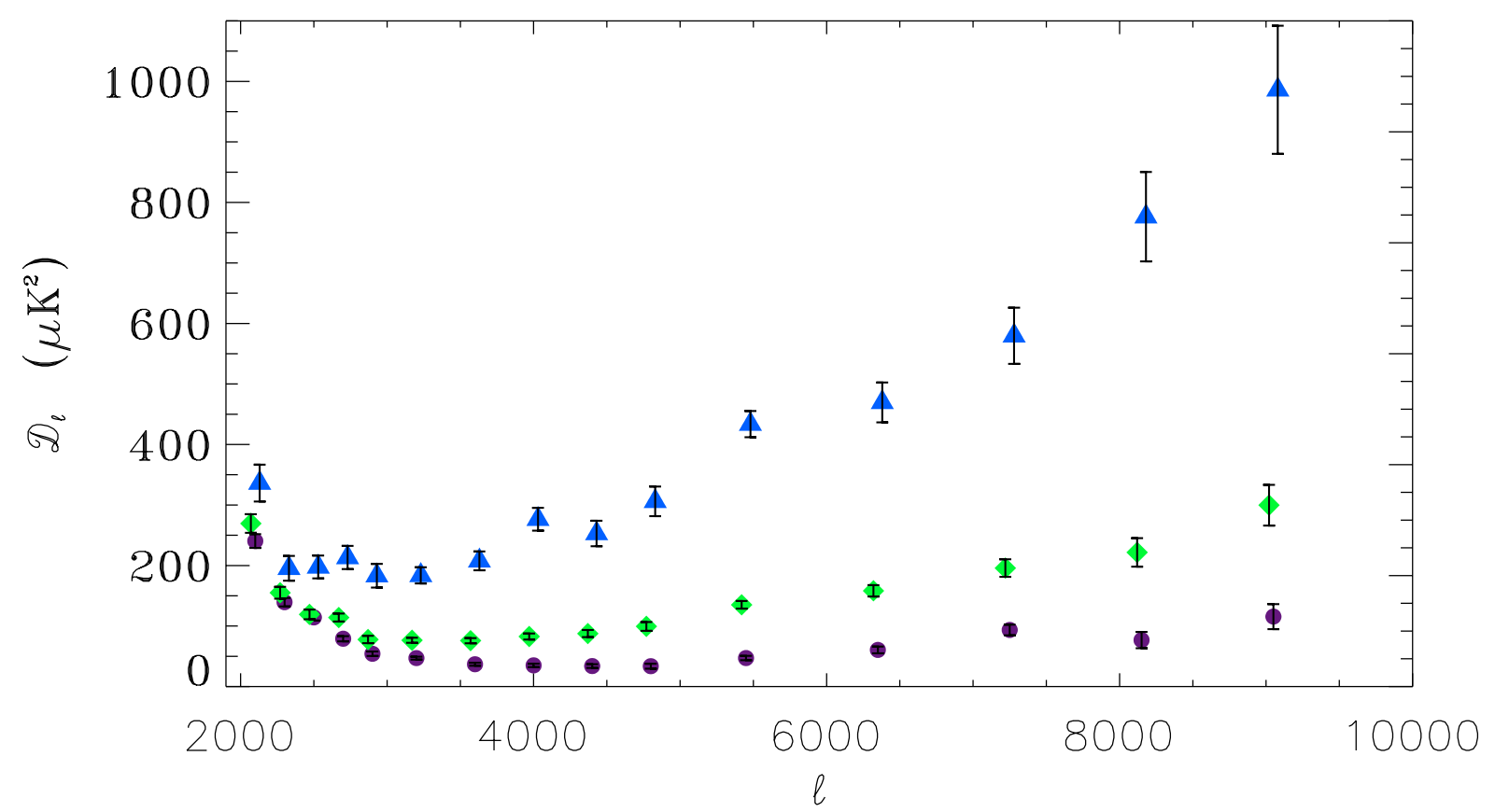

FIG. 3. - The SPT $150 \mathrm{GHz}$ (purple circles), $150 \times 220 \mathrm{GHz}$ (green diamonds) and $220 \mathrm{GHz}$ (blue triangles) bandpowers. The damping tail of the primary CMB anisotropy is apparent below $\ell=3000$. Above $\ell=3000$, there is a clear excess with an angular dependence consistent with point sources. These sources have low flux (as sources with > $7.4 \mathrm{mJy}$ at $150 \mathrm{GHz}$ have been masked) and a rising frequency spectrum, consistent with our expectations for Poisson distributed DSFGs. The point source population and resulting contributions to anisotropy power are discussed in more detail in H09.

TABLE 1

SINGLE-FREQUENCY BANDPOWERS

\begin{tabular}{cc|cc|cc|cc}
\hline \hline & & \multicolumn{2}{|c}{$150 \mathrm{GHz}$} & \multicolumn{2}{c}{$150 \times 220 \mathrm{GHz}$} & \multicolumn{2}{c}{$220 \mathrm{GHz}$} \\
$\ell$ range & $l_{\text {eff }}$ & $q\left(\mu \mathrm{K}^{2}\right)$ & $\sigma\left(\mu \mathrm{K}^{2}\right)$ & $q\left(\mu \mathrm{K}^{2}\right)$ & $\sigma\left(\mu \mathrm{K}^{2}\right)$ & $q\left(\mu \mathrm{K}^{2}\right)$ & $\sigma\left(\mu \mathrm{K}^{2}\right)$ \\
\hline $2001-2200$ & 2058 & 240.5 & 11.4 & 269.5 & 15.3 & 336.2 & 30.4 \\
$2201-2400$ & 2276 & 139.4 & 7.0 & 155.0 & 9.8 & 195.4 & 20.5 \\
$2401-2600$ & 2474 & 114.3 & 5.2 & 119.2 & 8.1 & 197.6 & 18.8 \\
$2601-2800$ & 2677 & 79.0 & 4.3 & 114.0 & 6.7 & 213.3 & 19.2 \\
$2801-3000$ & 2893 & 54.1 & 3.7 & 77.7 & 6.1 & 183.1 & 19.5 \\
$3001-3400$ & 3184 & 47.0 & 2.4 & 76.5 & 4.2 & 183.7 & 13.4 \\
$3401-3800$ & 3581 & 36.9 & 2.4 & 75.8 & 4.3 & 207.7 & 15.6 \\
$3801-4200$ & 3992 & 35.0 & 2.8 & 82.7 & 5.0 & 276.5 & 18.8 \\
$4201-4600$ & 4401 & 33.9 & 3.2 & 87.5 & 5.9 & 252.8 & 21.0 \\
$4601-5000$ & 4789 & 33.6 & 4.2 & 99.4 & 7.3 & 306.1 & 24.5 \\
$5001-5900$ & 5448 & 47.1 & 3.5 & 135.1 & 6.3 & 433.6 & 21.7 \\
$5901-6800$ & 6359 & 60.5 & 5.5 & 158.2 & 9.4 & 469.6 & 33.0 \\
$6801-7700$ & 7255 & 93.5 & 8.8 & 195.8 & 14.5 & 579.7 & 46.4 \\
$7701-8600$ & 8161 & 76.8 & 13.5 & 221.8 & 23.6 & 776.5 & 73.9 \\
$8601-9500$ & 9059 & 115.6 & 20.7 & 299.7 & 33.7 & 986.1 & 105.9 \\
\hline
\end{tabular}

Note. - Band multipole range and weighted value $\ell_{\text {eff }}$, bandpower $q_{B}$, and uncertainty $\sigma_{B}$ for the $150 \mathrm{GHz}$ auto-spectrum, cross-spectrum, and $220 \mathrm{GHz}$ auto-spectrum of the SPT field. The quoted uncertainties include instrumental noise and the Gaussian sample variance of the primary CMB and the point source foregrounds. The sample variance of the SZ effect, beam uncertainty, and calibration uncertainty is not included. Beam uncertainties are shown in Figure 1 and calibration uncertainties are quoted in 82.2 Point sources above $6.4 \mathrm{mJy}$ at $150 \mathrm{GHz}$ have been masked out in this analysis. This flux cut substantially reduces the contribution of radio sources to the bandpowers, although DSFGs below this threshold contribute significantly to the bandpowers.

We examine the residual Poisson point source amplitudes for a broad range of weighting ratios to estimate the optimal $x$ value and the error in that estimate. For each $x$, we estimate the probability that $C_{\ell}^{\mathrm{ps}}(x)$ is less than $C_{\ell}^{\mathrm{ps}}(x=0.325)$ using the MCMC chains described in 86.3 . The resulting probability distribution is taken to be the likelihood function for $x_{\text {true }}$. As shown in Figure 6. there is a broad maximum for $x=0.25$ to 0.4 and we adopt the best fit, $x=0.325$, for the following results. In the absence of a direct measurement, we place a con- 
TABLE 2

DSFG-SUBTRACTED BANDPOWERS

\begin{tabular}{cccc}
\hline \hline$\ell$ range & $l_{\text {eff }}$ & $q\left(\mu \mathrm{K}^{2}\right)$ & $\sigma\left(\mu \mathrm{K}^{2}\right)$ \\
\hline $2001-2200$ & 2058 & 221.3 & 16.9 \\
$2201-2400$ & 2276 & 130.2 & 11.2 \\
$2401-2600$ & 2474 & 126.5 & 10.3 \\
$2601-2800$ & 2677 & 60.2 & 7.7 \\
$2801-3000$ & 2893 & 50.4 & 8.0 \\
$3001-3400$ & 3184 & 36.6 & 5.9 \\
$3401-3800$ & 3581 & 21.0 & 6.5 \\
$3801-4200$ & 3992 & 22.9 & 8.4 \\
$4201-4600$ & 4401 & 8.1 & 9.5 \\
$4601-5000$ & 4789 & 3.0 & 11.3 \\
$5001-5900$ & 5448 & 11.2 & 10.5 \\
$5901-6800$ & 6359 & 16.0 & 16.2 \\
$6801-7700$ & 7255 & 60.3 & 27.9 \\
$7701-8600$ & 8161 & 32.1 & 42.9 \\
$8601-9500$ & 9059 & 54.7 & 63.8 \\
\hline
\end{tabular}

Note. - Band multipole range and weighted value $\ell_{\mathrm{eff}}$, bandpower $q_{B}$, and uncertainty $\sigma_{B}$ for the DSFG-subtracted maps of the SPT field. These bandpowers correspond to a linear combination (see 3.2 .4 ) of the $150,150 \times 220$, and $220 \mathrm{GHz}$ power spectra, optimized to remove emission from DSFGs below the point source detection threshold of SPT. Point sources above $6.4 \mathrm{mJy}$ at $150 \mathrm{GHz}$ have been masked out in this analysis. The quoted uncertainties include instrumental noise and Gaussian sample variance of the primary CMB and point source foregrounds. The sample variance of the SZ effect, beam uncertainty and calibration uncertainty is not included. Beam and calibration uncertainties are quoted in $\$ 2.1$ and 2.2 and shown in Figure 5

servative uniform prior on the scatter in DSFG spectral indices, $0.2<\sigma_{\alpha}<0.7$, as discussed in H09.

Our expectation for the residual radio contribution to the DSFG-subtracted bandpowers, $C_{\ell}^{\text {radio }}$, is based on the de Zotti et al. (2005) radio source count model. This model is in excellent agreement on the high flux end with the SPT source counts (V09). As discussed in H09, the residual radio source power after masking is expected to be a small fraction of the DSFG power at $150 \mathrm{GHz}$. However, this small radio contribution may be comparable to the residual DSFG power in the DSFG-subtracted spectrum. The radio source power can be calculated from the integral of $S^{2} d N / d S$ for the de Zotti et al. (2005) counts model from zero to the flux masking threshold of $6.4 \mathrm{mJy}$. We compute the power these sources contribute to the optimal DSFG-subtracted spectrum to be $3.9 \times 10^{-7} \mu \mathrm{K}^{2}$ by assuming an average spectral index of $\alpha=-0.5$ based on the detected sources in V09. This power level is nearly identical to that predicted by the Sehgal et al. (2009) simulations. To allow for a variation in spectral index as well as uncertainty in the model normalization when extended to lower flux sources, we assign a conservative uncertainty of $50 \%$ on the predicted residual radio source power in the prior.

We combine this information to create a prior on the residual point source power in the DSFG-subtracted maps $C_{\ell}^{\mathrm{ps}}=C_{\ell}^{\mathrm{DSFG}}+C_{\ell}^{\text {radio }}$. This prior spans the range $C_{\ell}^{\mathrm{ps}} \in[3.5,9.0] \times 10^{-7} \mu \mathrm{K}^{2}$ at $68 \%$ confidence and [1.2, $13.9] \times 10^{-7} \mu \mathrm{K}^{2}$ at $95 \%$ confidence. The best-fit value of the residual Poisson component before applying the prior is $C_{\ell}^{\mathrm{ps}}$ (no prior) $=(6.2 \pm 6.4) \times 10^{-7} \mu \mathrm{K}^{2}$ and lies at the middle of our assumed prior range. The upper end of the $95 \%$ range is approximately $20 \%$ of the best-fit value of the Poisson point source power in the undifferenced $150 \mathrm{GHz}$ bandpowers. This suggests that we have subtracted over $80 \%$ of the point source power from the
$150 \mathrm{GHz}$ spectrum, with the residual point source power largely from radio sources. Without this prior on the Poisson point source amplitude, the uncertainty on the $A_{\mathrm{SZ}}$ detection presented in the next section would increase by $\sim 50 \%$.

\subsubsection{Residual Clustered Point Source Power}

We assume that the contribution of clustered point sources is insignificant in the DSFG-subtracted bandpowers. Using a combination of the SPT bandpowers at 150,220 , and $150 \times 220 \mathrm{GHz}$ as well as the DSFGsubtracted spectrum, H09 argue that the residual clustered DSFG component in the DSFG-subtracted bandpowers is less than $0.3 \mu \mathrm{K}^{2}$ at $95 \%$ confidence. This is several percent of the SZ power spectrum but negligible at the current detection significance of $\lesssim 3 \sigma$. We also argue in $\$ 5$ that clustered radio sources are negligible. Therefore, residual power from clustered point sources will not bias SZ constraints from the DSFG-subtracted bandpowers.

The above argument holds if the point sources are uncorrelated with the SZ signal. However, if the clustered term was completely anti-correlated with the SZ signal, the measured SZ power in Table 3 could underestimate the true SZ power by $38 \%$. This is unlikely for two reasons. First, the residual after DSFG subtraction should be uncorrelated as long as the spectral dependence of cluster member DSFGs is similar to the general DSFG population. Second, as argued in $\$ 5$, most DSFGs are not galaxy cluster members. We also look at the correlations between the Sehgal et al. (2009) DSFG and tSZ simulated sky maps. The Sehgal et al. (2009) DSFG model scales the number density of DSFG cluster members linearly with cluster mass; this is a substantially stronger scaling than observed (Bai et al. 2007). Therefore, estimating the cluster-DSFG correlation from the 


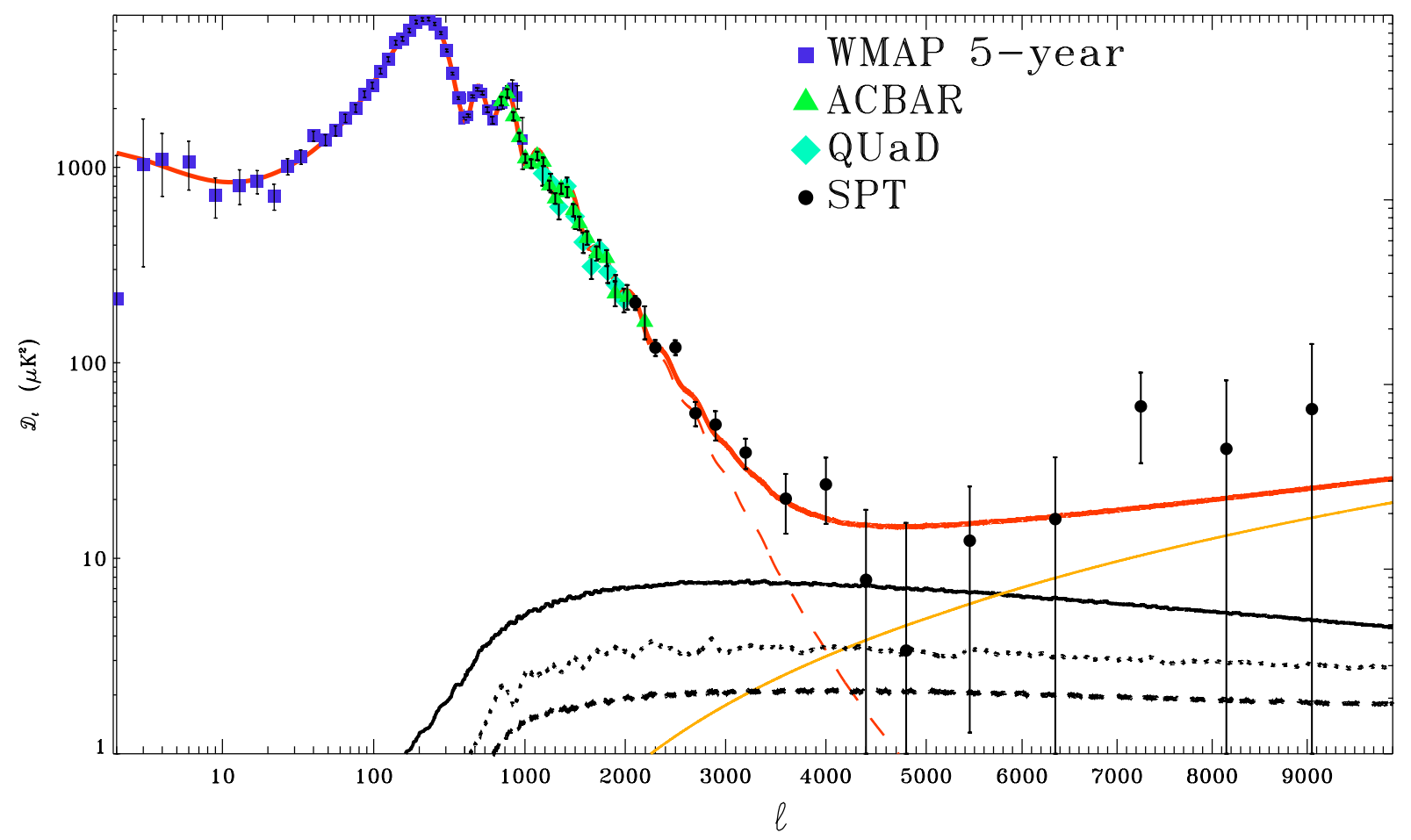

FIG. 4.- WMAP5 (blue squares), ACBAR (green triangles), QUaD (turquoise diamonds) and the SPT (black circles) DSFGsubtracted SPT bandpowers are plotted over the best-fit, lensed $\Lambda$ CDM cosmological model (dashed red line), best-fit tSZ power spectrum (solid black line), homogeneous kSZ model (dashed black line), and residual Poisson-distributed point source contribution (solid orange line). The combined best-fit model is shown by the solid red line. The plotted SPT bandpowers have been multiplied by the best-fit calibration factor of 0.92 . Point sources above $6.4 \mathrm{mJy}$ at $150 \mathrm{GHz}$ have been masked. The patchy kSZ template is also shown for reference (dotted black line). The DSFG-subtracted bandpowers are normalized to preserve the amplitude of the primary CMB anisotropies.

Sehgal et al. (2009) should be overly conservative. We calculate the anti-correlation coefficient between the Sehgal et al. (2009) simulated tSZ maps at $148 \mathrm{GHz}$ and a linear combination of the 148 and $219 \mathrm{GHz}$ simulated IR source maps with the same weighting as used for the SPT DSFG-subtracted bandpowers. We find the anticorrelation coefficient between the tSZ effect and total DSFG power to be $21 \%$ using the power measured at $\ell=3000$. This highly conservative upper limit of $21 \%$ on the anti-correlation implies that the true SZ power is underestimated by less than $4 \%$. Based on these arguments, we assume that correlations between SZ signals and emission from cluster member galaxies is negligible in this analysis.

Separately, one might worry about correlations between radio sources and SZ clusters. Radio sources could suppress the tSZ signal by "filling in" the tSZ decrements. However, the work by Lin et al. (2009) and Sehgal et al. (2009) shows the number of cluster-correlated radio sources is expected to be small. For instance, we can examine this correlation in the simulated tSZ and radio source maps produced by Sehgal et al. (2009). We look at the power spectrum at $\ell=3000$ for the tSZ map, the tSZ+radio-source map, and the radio source only map after masking sources above $6.4 \mathrm{mJy}$. We find an anti-correlation coefficient of $2.3 \%$ for the two components. Given the expected radio source power level of $\sim 0.6 \mu \mathrm{K}^{2}$ at $\ell=3000(6.2 .1$, radio source-cluster correlations should not affect the results in this work.

\subsection{Markov Chain Analysis}

The DSFG-subtracted bandpowers presented in 86.2 detect at high significance a combination of the primary $\mathrm{CMB}$ anisotropy, secondary $\mathrm{SZ}$ anisotropies, and residual point sources. In this section, we use an MCMC analysis to separate these three components and to produce an unbiased measurement of the tSZ power spectrum amplitude.

\subsubsection{Elements of the MCMC Analysis}

We fit the DSFG-subtracted bandpowers to a model including the lensed primary CMB anisotropy, secondary tSZ and kSZ anisotropies, and a residual Poisson point source term. We use the standard, six-parameter, spatially flat, lensed $\Lambda \mathrm{CDM}$ cosmological model to predict the primary CMB temperature anisotropy. The six parameters are the baryon density $\Omega_{b}$, the density of cold dark matter $\Omega_{c}$, the optical depth to recombination $\tau$, the angular scale of the peaks $\Theta$, the amplitude of the primordial density fluctuations $\ln \left[10^{10} A_{s}\right]$, and the scalar spectral index $n_{s}$. To fit the high- $\ell$ power, we extend the basic, six-parameter model with two additional parameters: the amplitude of a tSZ power spectrum template, $A_{\mathrm{SZ}}$, and a constant, $C_{\ell}^{\mathrm{ps}}$, such as would be produced by a Poisson distribution of point sources on the sky. We also explore the potential impact of the $\mathrm{kSZ}$ effect on these parameters by using three different kSZ models.

Gravitational lensing of CMB anisotropy by large scale structure tends to increase the power at small angular scales, with the potential to influence a SZ power spectrum measurement. The calculation of lensed CMB spectra out to $\ell=10000$ proved prohibitively expensive in 


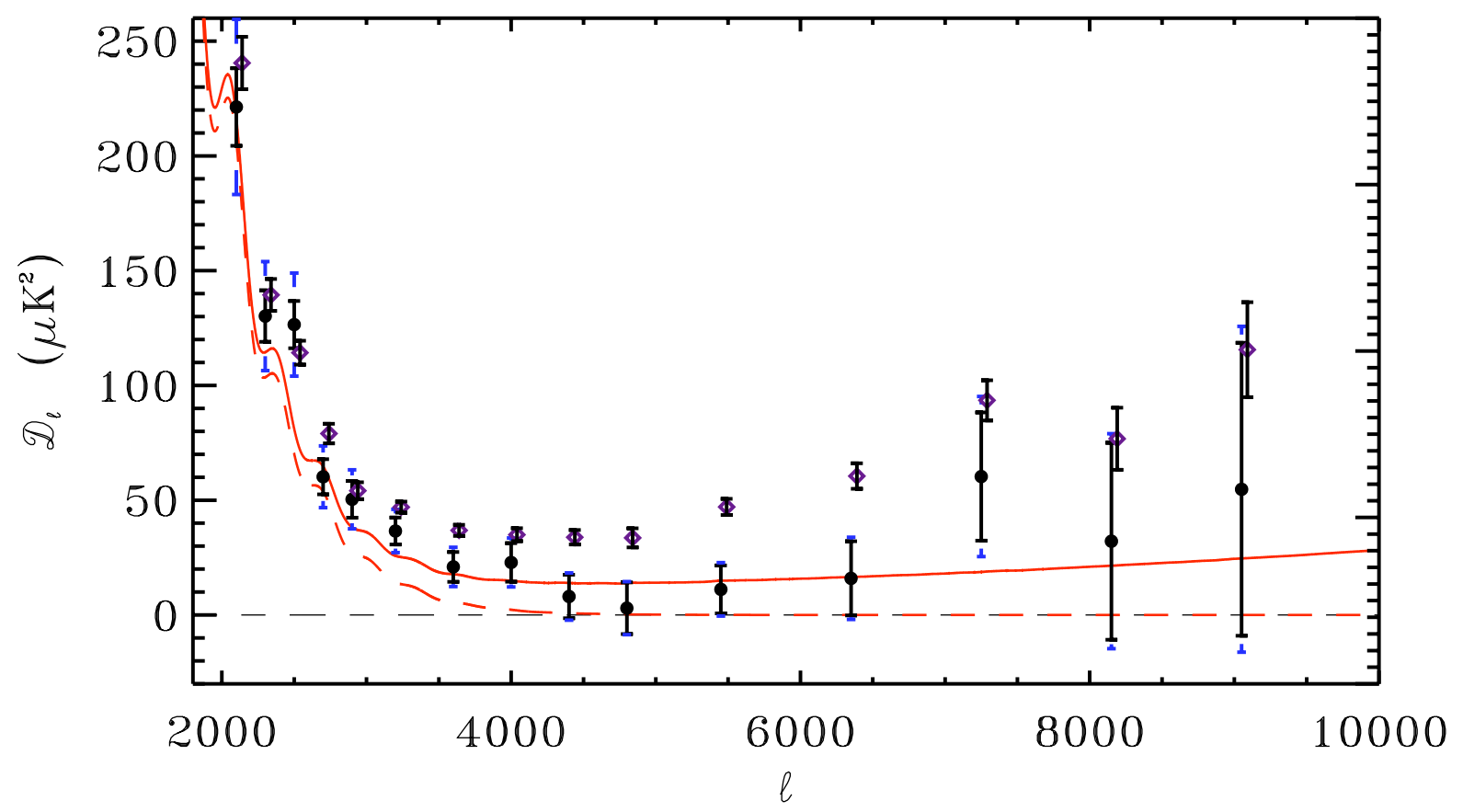

FIG. 5. - The SPT $150 \mathrm{GHz}$ (purple diamonds) and DSFG-subtracted (black circles) bandpowers over-plotted on the best-fit models to the DSFG-subtracted bandpowers. The best-fit, lensed $\Lambda$ CDM cosmological model for the primary CMB anisotropy is shown by the dashed red line, while the sum of the best-fit $\Lambda \mathrm{CDM}$ model, kSZ, tSZ and point source terms is shown by the solid red line. The primary CMB anisotropy alone is a poor fit to the SPT data. The uncertainties on the DSFG-subtracted bandpowers are larger for two reasons. First, the normalization convention inflates the uncertainties by a factor of $1 / 0.675^{2}$, and second, these bandpowers also include the more noisy $220 \mathrm{GHz}$ data. Beam and calibration uncertainties are marked by a second blue error bar for the DSFG-subtracted bandpowers only. Note that the calibration and beam uncertainties are correlated between $\ell$-bins. The $150 \mathrm{GHz}$ data has been shifted to the right by $\Delta \ell=40$ for clarity. Point sources above $6.4 \mathrm{mJy}$ at $150 \mathrm{GHz}$ have been masked in this analysis.

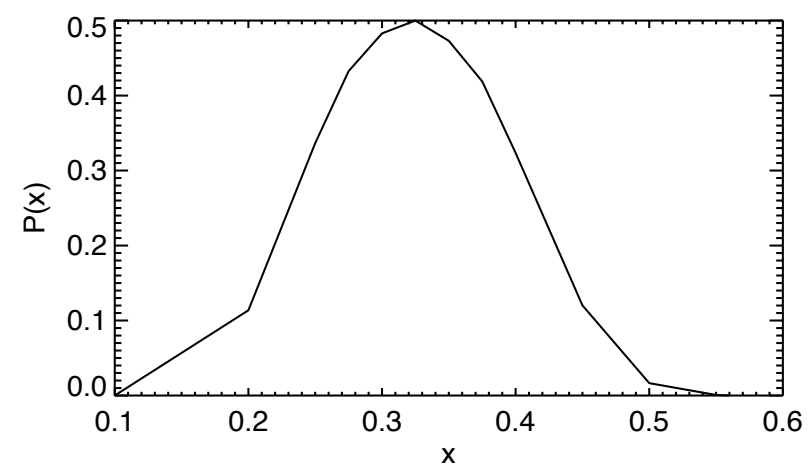

FIG. 6.- Probability that the residual point source power in the DSFG-subtracted map constructed by $\bar{m}_{150}-x \bar{m}_{220}$ is lower than the value at $x=0.325$ as a function of $x$. We can interpret this as the probability that a given value of $x$ is the true value. There is a broad maximum centered at 0.325 , which corresponds to a spectral index of 3.6 between the 150 and $220 \mathrm{GHz}$ bands. This is consistent with the ratio of point source power between single-frequency fits to the $150,150 \times 220$, and $220 \mathrm{GHz}$ bandpowers. We estimate the $x$ which minimizes residual point source power to be $0.325 \pm 0.08$.

computational time. We avoid this computational limitation by calculating the lensing contribution for the the best-fit cosmological model, and adding this estimated lensing contribution to the unlensed CMB power spectrum calculated at each step in the Markov chain. Given the small allowed range of $\Omega_{m}$ with current CMB data, we predict that using the fixed lensing contribution will misestimate the actual lensing by less than $30 \%$. We have checked this assertion on a sampling of parameter sets drawn from the chains. The lensing contribution to the high- $\ell$ spectrum is $\sim 1.5 \mu \mathrm{K}^{2}$ while the modeled tSZ spectrum for the same best-fit WMAP5 cosmology averages $\sim 8.6 \mu \mathrm{K}^{2}$ near $\ell=3000$ where SPT has the highest $\mathrm{S} / \mathrm{N}$ on the $\mathrm{SZ}$ spectrum. In the differenced spectra used to derive the $A_{\mathrm{SZ}}$ constraints (see 66.2 ), the ratio of lensing to the tSZ effect is suppressed by a factor of $(1-x)^{2}=0.46$. This reduction occurs because the subtracted spectrum is normalized such that the CMB power is unaffected, though the SZ power is enhanced. As a result, we expect possible lensing misestimates to introduce a negligible error of $\lesssim 3 \%$ on the $A_{\mathrm{SZ}}$ constraints. Of course, this error will be larger for smaller values of $A_{\text {SZ. }}$.

The tSZ template we use as a fiducial model is based on simulations by Sehgal et al. (2009). ${ }^{26}$ The simulations are for a WMAP5 cosmology with $\sigma_{8}=0.80$ and $\Omega_{b} h=0.0312$ and an observing frequency of $148 \mathrm{GHz}$. We rescale the template to $153 \mathrm{GHz}$, the effective center frequency of the SPT $150 \mathrm{GHz}$ band for signals with a tSZ spectrum.

For a limited number of cases, we also compare the results of the Sehgal numerical tSZ template to those of the Komatsu \& Seliak analytic template with WMAP5 parameters $^{27}$ (Komatsu \& Seljak 2002) and the numerical template by Shaw et al. (2009). The primary difference between the Shaw and Sehgal simulations is the value of the energy feedback parameter in the Bode et al. (2007) intracluster gas model. The Shaw simulations have the feedback parameter reduced to $60 \%$ of the Sehgal value. Increasing the feedback parameter causes the gas distri-

\footnotetext{
${ }^{26}$ http://lambda.gsfc.nasa.gov/toolbox/tb_cmbsim_ov.cfm

${ }^{27}$ http://lambda.gsfc.nasa.gov/product/map/dr3/pow_sz_spec_get.cfm
} 
bution in clusters to 'puff-out' or inflate; in low mass clusters the gas may become unbound altogether. The overall effect is to reduce the predicted tSZ power spectrum, especially at small angular scales. We limit our analysis of specific tSZ models to these three models.

An independent measurement of the $\mathrm{kSZ}$ spectrum is outside the scope of this work, however, it is necessary to take the $\mathrm{kSZ}$ effect into account when inferring the $\mathrm{tSZ}$ amplitude from the data. We consider three kSZ cases based on two published $\mathrm{kSZ}$ models. The $\mathrm{kSZ}$ effect is assumed to be zero in the first case ("no kSZ"). As a second, intermediate case, we use the model by Sehgal et al. (2009) (referred to as "homogeneous kSZ"). This model includes $\mathrm{kSZ}$ contributions from a homogeneous reionization scenario, but does not include the additional $\mathrm{kSZ}$ power produced by patchy reionization scenarios. We take this case to be the fiducial $\mathrm{kSZ}$ model. We include an estimate of patchy reionization in the third $\mathrm{kSZ}$ case. For the patchy reionization phase, we use the "brief history" model B from Zahn et al. (2005), re-calculated for WMAP-5 best fit cosmological parameters. The sum of the homogeneous $\mathrm{kSZ}$ model and the patchy contribution will be referred to as the "patchy kSZ" model. We do not scale these templates for different cosmological parameter sets as we expect the kSZ theoretical uncertainty to be at least as large as the cosmological dependence. Finally, we include a residual Poisson point source component in all chains. The construction of the point source prior is outlined in 6.2 .1 .

Previous CMB experiments have produced exceptional constraints on the primary CMB anisotropy, and we use the bandpowers from WMAP5 (Dunkley et al. 2009), ACBAR (Reichardt et al. 2009a), and QUaD (Brown et al. 2009) at $\ell<2200$ in all parameter fitting. We refer to this collection along with the SPT DSFG-subtracted bandpowers as the 'CMBall' data set. It is important to note that the two-parameter extension to the $\Lambda \mathrm{CDM}$ model for point sources and the tSZ effect is only restricted to the SPT bandpowers. This restriction is imposed for two reasons. First, the point source contributions to each experiment may be different due to the different frequencies and flux cuts for masking sources. Arguably, the point source power is likely to be similar in the $150 \mathrm{GHz}$ SPT, ACBAR, and QUaD results, but we would be unable to use frequency information to discriminate between the SZ effect and clustered DSFGs. Second, the primary CMB is dominant below $\ell \sim 3000$ and the other experiments lack sufficient statistical weight at high- $\ell$ to improve upon SPT's measurement of SZ effect and point source power.

Parameter estimation is performed by MCMC sampling of the full multi-dimensional parameter space using an extension of the CosmoMC package (Lewis \& Bridle 2002). We include the code extension produced by the QUaD collaboration (Brown et al. 2009) to handle uncertainties on a non-Gaussian beam in CosmoMC. CMB power spectra for a given parameter set are calculated with CAMB (Lewis et al. 2000). We use the WMAP5 likelihood code publicly available from http://lambda.gsfc.nasa.gov, After the burn-in period, each set of four chains is run until the largest eigenvalue of the Gelman-Rubin test is smaller than 0.0005 . Wide uniform priors are used on all six parameters of the $\Lambda \mathrm{CDM}$ model. A weak prior on the age of the Universe $\left(t_{0} \in[10,20]\right.$ Gyrs $)$ and Hubble constant $(h \in[0.4,1])$ is included in all chains, but should not affect the results. We use a uniform prior on $A_{\mathrm{SZ}}$ over a wide range from -1 to 10 times the value expected for $\sigma_{8}=0.80$.

\subsubsection{Constraints on $S Z$ amplitude}

We fit for the normalization factor of a fixed tSZ template and, in Table 3 , report both this template-specific normalization, $A_{\mathrm{SZ}}$, and the total inferred SZ-power at $\ell=3000$, near the multipole with maximum tSZ detection significance. This estimate of the SZ power includes both tSZ and kSZ terms, and is included to facilitate comparison with other SZ models. We expect both the thermal and the kinetic SZ spectra to vary slowly with angular multipole.

The chains are run for three different assumptions about the kSZ effect: the no kSZ, homogeneous kSZ, and patchy $\mathrm{kSZ}$ models described above. In each case, we produce MCMC chains with a fixed $\mathrm{kSZ}$ amplitude. The $\chi^{2}$ of the 15 SPT bandpowers is between 16.3 and 16.4 for the best fits of the three $\mathrm{kSZ}$ cases considered; there is essentially no impact on the quality of the fit. The $A_{\mathrm{SZ}}$ and power constraints for each case are listed in Table 3 and plotted in Figure 7 .

The bandpower uncertainties in Table 2 do not include the sample variance of the tSZ effect, and we must convolve the $A_{\mathrm{SZ}}$ distribution in the chains with an estimate of the sample variance to find the true $A_{\mathrm{SZ}}$ likelihood function. The sample variance of the tSZ effect is estimated using the simulations of Shaw et al. (2009) rerun with the same intracluster gas model parameters as Sehgal et al. (2009). The simulation consists of 300 map realizations of the size of the SPT sky patch. These are constructed from a base sample of 40 independent maps by separating the components of each map into eight redshift bins (between $0 \leq z \leq 3$ ) and shuffling these bins between maps to generate a larger sample. We take the spectrum of each realization and find the bestfit $A_{\mathrm{SZ}}$ amplitude after weighting $\ell$-bins based on the SPT bandpower uncertainties. We use the distribution of these amplitudes to map out the likelihood function for the tSZ power (see Figure 8). Due to the number of independent realizations, we have limited ability to resolve the tail of the likelihood function. Fortunately, the non-Gaussianity is small for such a large sky area and the distribution of $\ln \left(A_{\mathrm{SZ}}\right)$ is well-fit by a Gaussian with a $12 \%$ width. We use this Gaussian fit as an estimate of the full likelihood surface. Small deviations from the true description of the tSZ sample variance will not impact the final results as the sample variance is small compared to both the statistical uncertainties and the assumed $50 \%$ model uncertainty. The uncertainties on $A_{\mathrm{SZ}}$ are essentially unchanged by the inclusion of the tSZ sample variance. The sample variance and model uncertainty are shown in Figure 8.

As discussed earlier, the DSFG-subtracted bandpowers are sensitive to a linear combination of the $\mathrm{tSZ}$ and $\mathrm{kSZ}$ effects. We expect analysis of 2009 and later SPT data which include $95 \mathrm{GHz}$ data to be able to separate the two SZ effects. The linear combination is not a simple sum, as the frequency-differencing used to produce the DSFG-subtracted spectrum suppresses the $\mathrm{kSZ}$ relative to the tSZ by a factor of $(1-x)^{2}=0.46$. This factor is uncertain at the $15 \%$ level due to the relative cali- 
TABLE 3

Constraints on $A_{\mathrm{SZ}}$ AND $\sigma_{8}$

\begin{tabular}{lcc}
\hline \hline & primary CMB & $\mathrm{CMB}+A_{\mathrm{SZ}}:$ \\
\hline$A_{\mathrm{SZ}}:$ & - & $0.55 \pm 0.21$ \\
$A_{\mathrm{SZ}}($ w homogeneous kSZ): & - & $0.42 \pm 0.21$ \\
$A_{\mathrm{SZ}}$ (w patchy kSZ): & - & $0.34 \pm 0.21$ \\
\hline SZ power at $\ell=3000$ & & \\
( tSZ $+0.46 \times \mathrm{kSZ}):$ & - & $4.2 \pm 1.5 \mu \mathrm{K}^{2}$ \\
\hline kSZ power at $\ell=3000$ & & \\
$\quad$ homogeneous kSZ & & $2.0 \mu \mathrm{K}^{2}$ \\
$\quad$ patchy kSZ & & $3.3 \mu \mathrm{K}^{2}$ \\
\hline$\sigma_{8}($ no kSZ): & $0.795 \pm 0.033$ & $0.778 \pm 0.024$ \\
$\sigma_{8}($ w homogeneous kSZ): & $0.794 \pm 0.028$ & $0.773 \pm 0.025$ \\
$\sigma_{8}($ w patchy kSZ): & $0.788 \pm 0.029$ & $0.770 \pm 0.024$ \\
\hline \hline
\end{tabular}

Note. - The $1 \sigma$ constraints on $\sigma_{8}$ derived from the DSFG-subtracted analysis of the SPT data, when using the simulations in Shaw et al. (2009) to estimate the non-Gaussian cosmic variance of the tSZ power spectrum. The best-fit value for the amplitude of the tSZ power spectrum is also shown, normalized to unity for a WMAP5 cosmology with $\sigma_{8}=0.8$. $A_{\mathrm{SZ}}=1$ corresponds to a power of $7.5 \mu \mathrm{K}^{2}$ at $\ell=3000$. Results are shown with cosmic variance added in quadrature to the statistical uncertainty, however, the $\mathrm{A}_{\mathrm{SZ}}$ constraints are dominated by statistical uncertainties. Results are shown for no kSZ effect, for a homogeneous model of the kSZ effect (Sehgal et al. 2009) and the homogeneous model with an additional patchy reionization power contribution (Zahn et al. 2005). Finally, the joint constraint on the combined kSZ/tSZ power is shown under the assumption that the two templates are effectively degenerate. For reference, we also quote the power of the two kSZ models considered. The SPT data constrains the combined amplitude of the SZ contributions, which we quote at $\ell=3000$ where the measurements have the most constraining power. The $\mathrm{kSZ}$ and $\mathrm{tSZ}$ receive different pre-factors in the frequency-differenced analysis due to their relative spectral dependence.

bration uncertainty between the bands. The SPT data detect the combined SZ effect at $2.6 \sigma$ with tSZ +0.46 $\times \mathrm{kSZ}=4.2 \pm 1.5 \mu \mathrm{K}^{2}$ at $\ell=3000$. Using this combined constraint implicitly assumes that the tSZ and kSZ templates are perfectly degenerate, which is a good assumption for the current data quality and templates used in this work.

We can compare the power detected with SPT to that reported by the CBI collaboration (Sievers et al. 2009). We use the best-fit normalization of a Komatsu \& Seljak template for WMAP5 parameters to compare directly the results of the two experiments. There are sub-percent differences in the assumed values of $\sigma_{8}$ and $\Omega_{b} h$ between the template we adopted here and that used in Sievers et al. (2009), which would change the amplitude of the template by $\sim 1 \%$ for an assumed scaling of $\sigma_{8}^{7}\left(\Omega_{b} h\right)^{2}$. This effect is negligible. We find the best-fit normalization of the WMAP5 Komatsu \& Seljak model to be $0.37 \pm 0.17$ for the SPT data under the homogeneous $\mathrm{kSZ}$ scenario. This is $2.4 \sigma$ below the best-fit CBI normalization of $3.5 \pm 1.3$. The model includes the frequency dependence of the tSZ effect. The smaller SPT bandpowers suggest that the CBI excess power may be produced by foregrounds with a frequency dependence falling more steeply than the SZ effect such as radio sources.

\subsection{Implications of the $A_{\mathrm{SZ}}$ Measurement}

The best-fit normalization for the fiducial tSZ spectrum, $A_{\mathrm{SZ}}$, is significantly lower than unity. The cosmological parameters assumed when generating this template may be slightly different than the best-fit models, so we scale the template to match the cosmologies explored by the Markov chain. When these scalings are taken into account, the measured values of $A_{\mathrm{SZ}}$ are still low. The tSZ power spectrum depends on the details of how the baryon intracluster gas populates dark matter halos and on cosmology through the number density of these halos. The paucity of tSZ power may reflect an overestimate of the intracluster gas pressure by the fiducial model and so we compare the template to other SZ models. At the same time, this low value of $A_{\mathrm{SZ}}$ favors a shift in the derived cosmological parameters, particularly $\sigma_{8}$

Even a $\sim 2.5 \sigma$ detection of $A_{\mathrm{SZ}}$ will produce cosmologically interesting constraints on $\sigma_{8}$, as we expect $A_{\mathrm{SZ}}$ to scale strongly with $\sigma_{8}$ and less strongly with the baryon density. The scaling is approximately $\sigma_{8}^{\gamma}\left(\Omega_{b} h\right)^{2}$ where $7<\gamma<9$, depending on the exact cosmology (Komatsu \& Kitayama 1999 Komatsu \& Seljak 2002). We explore the $\sigma_{8}$ dependence under the Press-Schechter halo model and find that this relationship steepens with the currently favored lower values of $\sigma_{8}$. Sampling cosmological parameter values from the WMAP5 MCMC chains (which properly treats degeneracies between $\sigma_{8}$ and other parameters), we find the modeled amplitude of the tSZ power spectrum varies approximately as $\sigma_{8}^{11}$. Constraining the amplitude of the tSZ effect offers an independent measurement of $\sigma_{8}$ that can be compared to measurements based on primary CMB anisotropy or large scale structure. Such comparisons test our understanding of the physical processes involved in structure formation.

For each point in the MCMC chain, we calculate the predicted $A_{\mathrm{SZ}}$ value from the six basic cosmological parameters. For this calculation, we use the mass function of Jenkins et al. (2001) to determine the abundance of galaxy clusters of a given mass. We then use the massconcentration relation of Duffy et al. (2008) to determine the dark matter halo properties, and the gas model used 


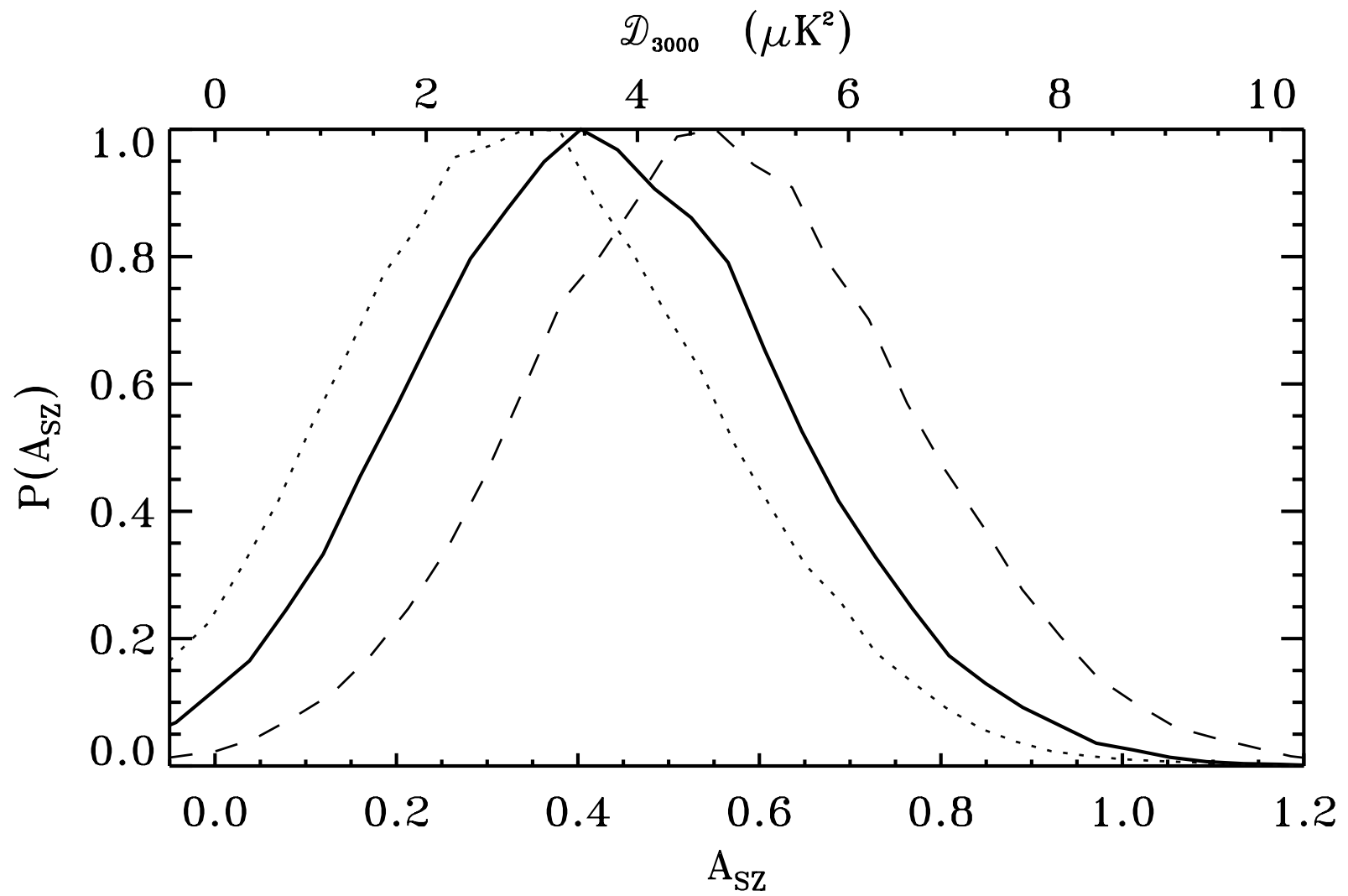

FIG. 7.- The 1D marginalized ASZ constraints from the SPT DSFG-subtracted bandpowers. Three kSZ cases are considered: no kSZ effect (dashed line), the homogeneous kSZ model (solid line) and the homogeneous model plus a patchy reionization term (dotted line). These models are described more fully in 86.3 .1 Top axis: The corresponding tSZ power at $\ell=3000$ for reference. The no-kSZ curve (dashed line) can be interpreted as a constraint on the sum of $D_{3000}^{\mathrm{tSZ}}+0.46 \times D_{\ell}^{\mathrm{kSZ}}$.

in Komatsu \& Seljak (2002) to estimate the tSZ signal for each halo according to its mass. In order to convert this analytic tSZ spectrum into an amplitude, we take an $\ell$-weighted average designed to match the relative weights each multipole receives in the real tSZ fits. This amplitude is normalized to unity for the cosmological parameters assumed in the fiducial tSZ model. We also allowed the mass-concentration index to vary with cosmology by appropriate scaling of the characteristic mass $M_{*}$, but this was found to be a negligible effect within the explored range in parameters.

At each point in the chain, the measured tSZ amplitude is compared to the predicted tSZ amplitude to construct a tSZ scaling factor, $\mathrm{A}_{\mathrm{SZ}} / \mathrm{A}_{\mathrm{SZ}}^{\text {theory }}$. This procedure will account for any correlations between the measured $\mathrm{A}_{\mathrm{SZ}}$ parameter and the six $\Lambda \mathrm{CDM}$ parameters in a selfconsistent fashion, although we do not see evidence for such correlations in the current data. The distribution of scaling factors vs. $\sigma_{8}$ is illustrated by the black contours in Figure 9 .

In general, the tSZ scaling factors are less than unity. These low scaling factors suggest either an over-estimate of the tSZ effect or lower values of $\sigma_{8}$. Models predicting larger $\mathrm{kSZ}$ or $\mathrm{tSZ}$ effects lead to lower scaling factors. However, the results can not be purely explained by an over-estimate of the $\mathrm{kSZ}$ effect since this tension persists in the no-kSZ case. Alternatively, the scaling factors may indicate that the explored range in cosmological parameters is systematically overestimating the RMS of the mass distribution. For instance, as we see in Figure 9 . points of the chain with lower values of $\sigma_{8}$, have scaling factors closer to unity.

The first interpretation of the low tSZ scaling factor is that the Sehgal tSZ template overestimates the tSZ power spectrum. There is currently some degree of uncertainty in the expected shape and amplitude of the tSZ power spectrum as predicted by analytic models or hydrodynamical simulations. One reason for this is that cosmological simulations of the intracluster medium have only recently begun to investigate in detail the impact of radiative cooling, non-gravitational heating sources (such as AGN), and possible regulatory mechanisms between them. The computational expense of running hydrodynamical simulations with sufficient resolution to resolve small-scale processes (such as star-formation) while encompassing a large enough volume to adequately sample the halo mass function is prohibitive to a detailed analysis of the predicted SZ power spectrum. In order to accurately predict the tSZ power spectrum, it is especially important to correctly model the gas temperature and density distribution in low mass $\left(M<2 \times 10^{14} h^{-1} M_{\odot}\right)$ and high redshift $(z>1)$ clusters, which contribute significantly to the power spectrum at the angular scales where SPT is most sensitive (Komatsu \& Seljak 2002).

In Figure 10, we plot the tSZ power spectrum derived from several different simulations (note that all curves have been normalized to the fiducial cosmology). The thick black solid line shows the base template, obtained from maps generated by Sehgal et al. (2009). The black dot-dashed line shows the power spectrum obtained from 


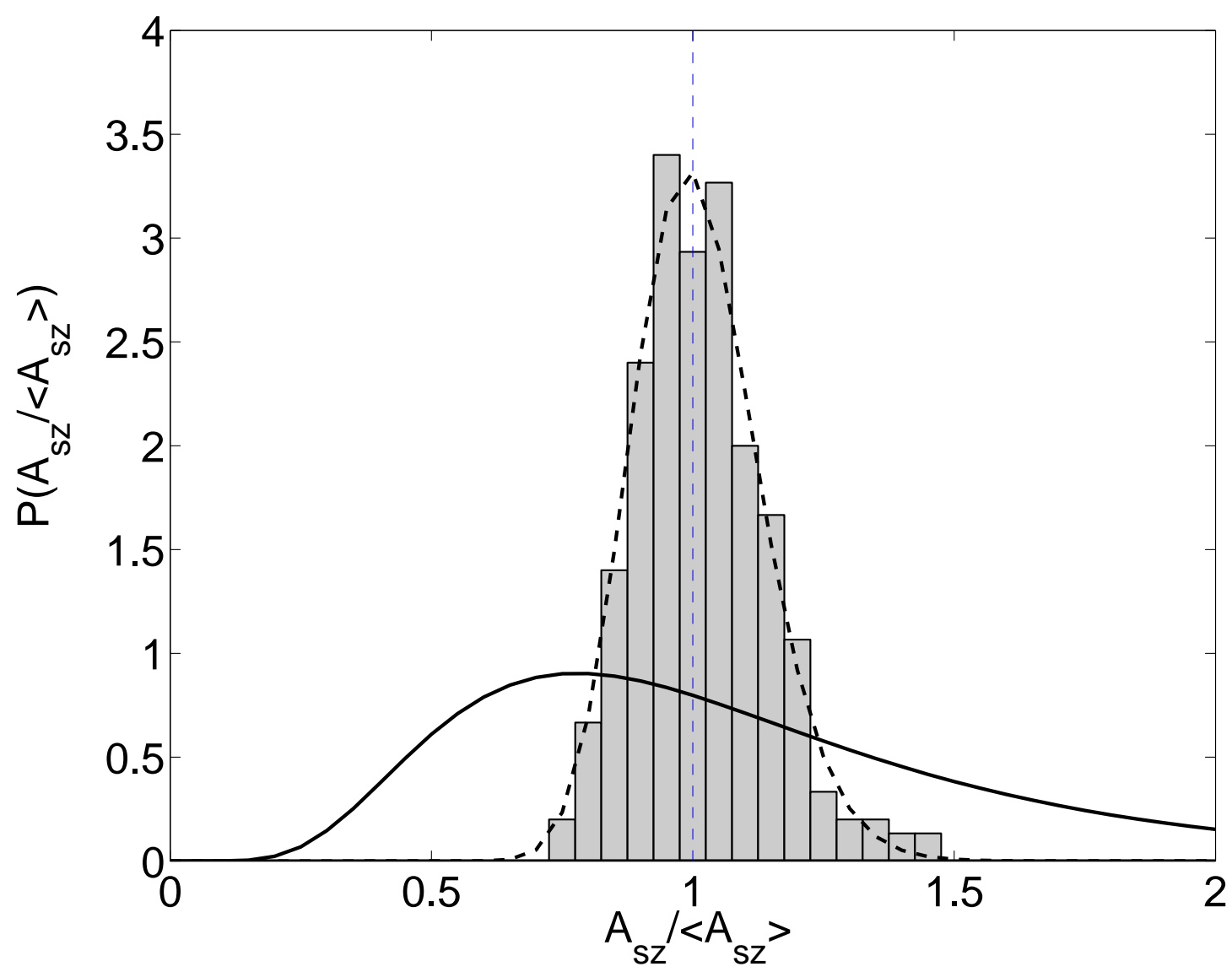

FIG. 8. - Sample variance and assumed theoretical uncertainty on the tSZ amplitude. The histogram shows the sample variance of $A_{\mathrm{SZ}} /\left\langle A_{\mathrm{SZ}}\right\rangle$, where $\left\langle A_{\mathrm{SZ}}\right\rangle$ is the mean value measured over a sample of 300 simulated maps. The overlying dashed black line shows the lognormal fit to the distribution, i.e., a Gaussian fit to $\ln \left(A_{\mathrm{SZ}}\right)$ ), with $\sigma_{\ln A}=0.12$. The solid black line is the assumed $50 \%$ theoretical uncertainty on $A_{\mathrm{SZ}}$, which we model as a Gaussian distribution in $\ln \left(A_{\mathrm{SZ}}\right)$.

the simulations of Shaw et al. (2009), which have a lower energy feedback parameter than the Sehgal et al. (2009) simulations. Increasing the amount of feedback energy has the effect of inflating the gas distribution and suppressing power at small angular scales. The red solid and dot-dashed lines show the power spectrum from maps constructed from a $240 h^{-1} \mathrm{Mpc}$ box simulation run using the Eulerian hydrodynamics code CART (Kravtsov et al. 2005, Douglas Rudd, private communication) and from the MareNostrum simulation (Zahn et al. 2010) respectively. Both of these simulations were run in the adiabatic regime, i.e., no cooling, star-formation or feedback. The gas distribution is more centrally concentrated than in the Shaw or Sehgal models, producing tSZ power spectra with significantly more power at small angular scales and less at larger angular scales. The blue solid line shows the analytic template predicted by the Komatsu \& Seljak (2002) halo model calculation. The histogram shows the SPT sensitivity to the thermal SZ signal in each $\ell$-band (with arbitrary normalization).

The current data are in tension with even the highfeedback simulations for the CMB-derived best-fit cosmological parameter set. Even with the kSZ effect set to zero, the tSZ scaling factor is only $0.55 \pm 0.21$ of what is predicted for the fiducial WMAP5 cosmology. Meanwhile, the tSZ scaling factors are $0.42 \pm 0.21$ for the ho- mogeneous kSZ model, and $0.34 \pm 0.21$ for the patchy $\mathrm{kSZ}$ model. The tension grows worse for the mediumfeedback simulations by Shaw et al. (2009). Although the Bode et al. (2007) model is calibrated to reproduce observed x-ray scaling relations for high-mass, low-redshift clusters, it may significantly over-estimate the contribution of low-mass or high-redshift clusters (for which there are few direct x-ray observations with which to compare).

As mentioned above, an alternate interpretation of the low tSZ scaling factor is that the CMBall parameter chains without SZ constraints overestimate $\sigma_{8}$. In order to constrain $\sigma_{8}$ based on the measurement of $\mathrm{A}_{\mathrm{SZ}}$, we need to construct the likelihood of observing $A_{\mathrm{SZ}}$ for a given cosmological parameter set. At a minimum, this likelihood would reflect the $12 \%$ uncertainty due to sample variance of the tSZ effect. However given the large range of $\mathrm{tSZ}$ predictions, we add an additional theory uncertainty in quadrature with the sample variance. The grey region in Figure 10 shows the $1 \sigma$ region encompassed by a lognormal distribution of width $\sigma_{A s z}=0.5$ around the fiducial model (black solid line). In the range where SPT is most sensitive $(2000 \leq \ell \leq 6000)$, all the predicted curves lie within this region. In order to account for the range of predicted power spectra, we adopt a $50 \%$ theory uncertainty on the value of $\ln \left(A_{s z}\right)$ in the likelihood calculation. The resulting $\sigma_{8}$ constraints are 

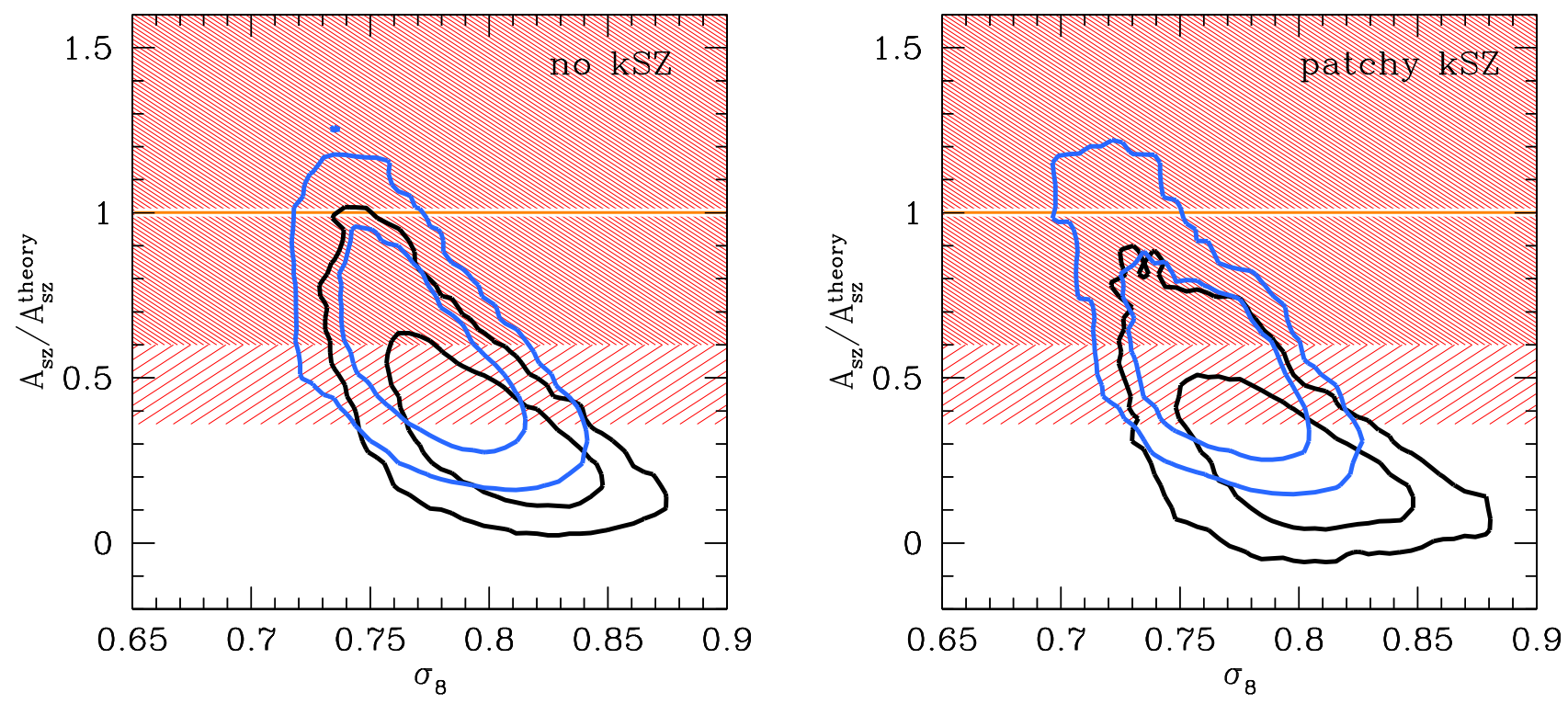

FIG. 9. Two-dimensional likelihood contours at $68 \%$ and $95 \%$ confidence for $\sigma_{8}$ versus the tSZ scaling factor, $A_{\mathrm{SZ}} / A_{\mathrm{SZ}}^{\text {theory }}$, derived from the SPT DSFG-subtracted bandpowers. For each point in the Markov chain, the tSZ scaling factor compares the $A_{\mathrm{SZ}}$ value fit to the SPT data to the $A_{\mathrm{SZ}}^{\text {theory }}$ value predicted for that point's $\Lambda \mathrm{CDM}$ model parameters (see 8.4 . The black contours show the likelihood surface for the CMBall dataset. We observe no dependence between $A_{S Z}$ and the six parameters of the $\Lambda$ CDM model. The tilt towards higher scaling factors at lower $\sigma_{8}$ is expected since the predicted $A_{\mathrm{SZ}}^{\text {theory }}$ depends steeply on the value of $\sigma_{8}$. The black contours also do not account for the cosmic variance of $A_{\mathrm{SZ}}$; without cosmic variance or uncertainty in modeling the tSZ power, the tSZ scaling factor would be constrained to be exactly unity (solid orange line). The red shaded regions about unity illustrate the uncertainty we assume for the tSZ scaling factor due to theoretical uncertainty and cosmic variance (also see Fig. 8). This uncertainty is modeled as a log-normal distribution. The measured value of the tSZ scaling relation, including the theoretical uncertainty and sample variance in the model, is used to importance sample the Markov chain and obtain the likelihood surface marked by the blue contours. Left panel: Likelihood surfaces assuming no kSZ contribution. Right panel: Likelihood surfaces assuming the patchy kSZ model. The constraints for the homogeneous $\mathrm{kSZ}$ model will lie between the results for these two cases.

dominated by the theory uncertainty. With this prior, we construct a new chain from the original parameter space through importance sampling. The regions preferred by this prior are shown in Figure 9, as are the results of the new Markov chain.

Constraints on $\sigma_{8}$ with and without including the SPT $A_{\mathrm{SZ}}$ measurements are shown in Table 3 and Figure 11. Under the assumption of the homogeneous kSZ model and a $50 \%$ theoretical uncertainty in the amplitude of the tSZ powerspectrum, the addition of the SPT data slightly tightens the constraint on $\sigma_{8}$, while reducing the central value from $\sigma_{8}=0.794 \pm 0.028$ to $0.773 \pm 0.025$. The uncertainty in the resulting constraint on $\sigma_{8}$ is dominated by the large theoretical uncertainty in the $\mathrm{tSZ}$ amplitude. If instead we assume that the fiducial tSZ model is perfectly accurate and do not account for model uncertainty, the uncertainty on $\sigma_{8}$ is reduced by $30 \%$ and the preferred value is significantly reduced to $\sigma_{8}=0.746 \pm 0.017$ for the homogenous kSZ model. Despite the fact that the adopted template is the lowest of the tSZ models shown in Figure 8, the Sehgal et al. (2009) template, the value of $\sigma_{8}$ inferred by the SPT data using this model is lower than that favored by WMAP. Additional SPT data will soon determine if the apparent tension between $\sigma_{8}$ inferred from SZ and primordial CMB measurements is robust. In any case, improving our theoretical understanding of both the kSZ and tSZ power spectra is essential for fully realizing the potential of SZ power spectrum measurements to constrain cosmological parameters such as $\sigma_{8}$

\section{CONCLUSIONS}

We have presented the first CMB temperature anisotropy power spectrum results from the SPT experiment. Table 1 contains bandpowers based on simultaneous observations at 150 and $220 \mathrm{GHz}$ of a single $100 \mathrm{deg}^{2}$ field for three months during the austral winter of 2008. A total of $286 \mathrm{k}$ detector-hours of data was collected during these observations, resulting in a final map depth of $18 \mu \mathrm{K}$-arcmin at $150 \mathrm{GHz}$ and $40 \mu \mathrm{K}$-arcmin at $220 \mathrm{GHz}$. The SPT $150 \mathrm{GHz}$ temperature maps are calibrated to an accuracy of $3.6 \%$ through a direct comparison of CMB temperature anisotropy as observed by SPT and WMAP5. This absolute temperature calibration is extended to $220 \mathrm{GHz}$ with a $7.2 \%$ uncertainty by an internal comparison of the primary CMB anisotropy measured by SPT at 150 and $220 \mathrm{GHz}$.

The SPT bandpowers include angular multipoles from $\ell=2000$ to 9500 . This broad angular range covers the transition from where the primary CMB anisotropy dominates the measured power to where secondary $\mathrm{CMB}$ anisotropies and foregrounds become dominant. The primary anisotropy observed by SPT is consistent with $\Lambda \mathrm{CDM}$ cosmological models based on lower- $\ell$ CMB data. At $\ell>3000$, the SPT bandpowers are used to detect an excess of power above the primary anisotropies at $\sim 50 \sigma$ at both 150 and $220 \mathrm{GHz}$ after masking bright $(>6.4 \mathrm{mJy})$ sources.

Most of this power can be explained by a population of 


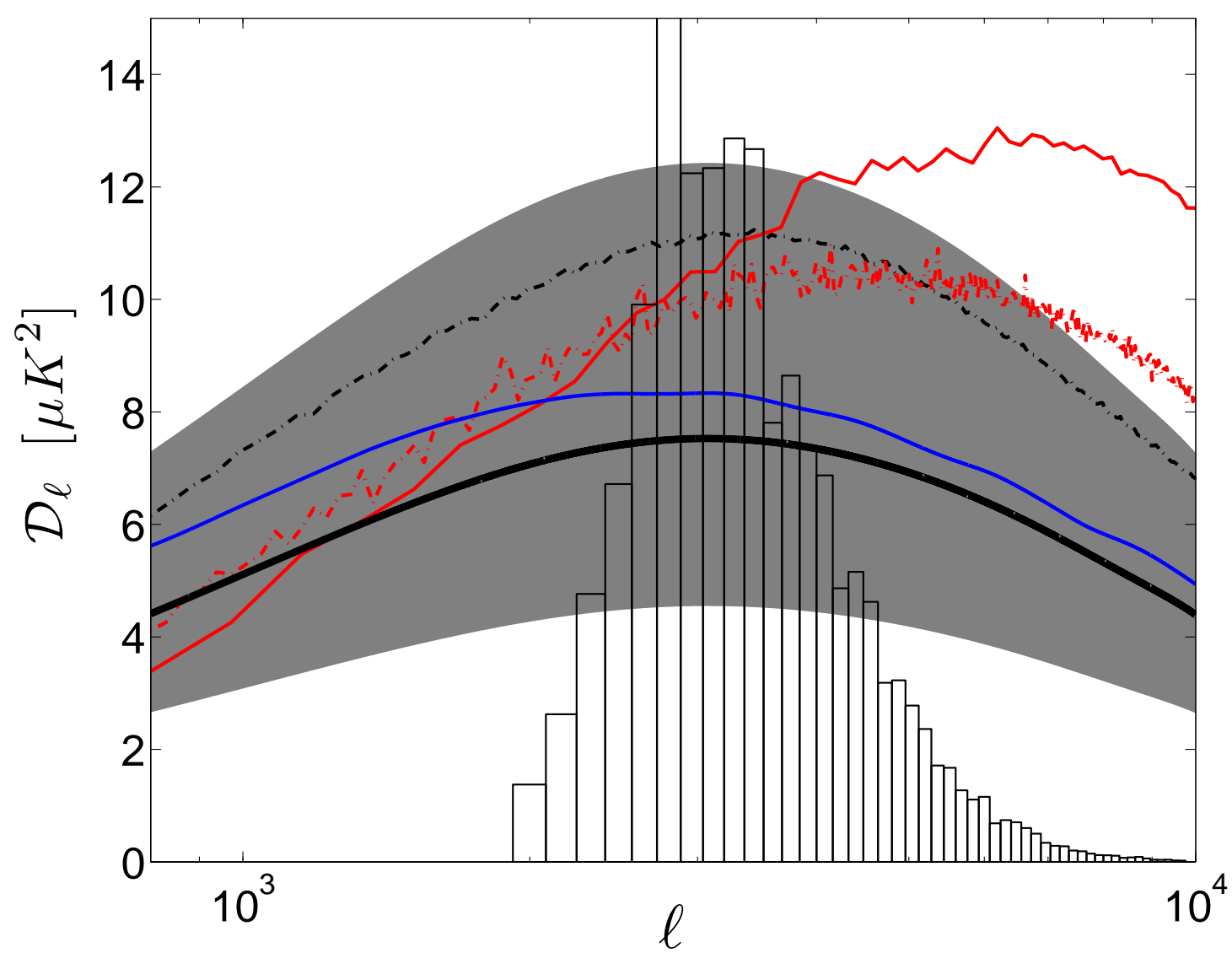

FIG. 10. - Comparison of the tSZ power spectrum (at $153 \mathrm{GHz}$ ) as predicted by numerical simulations and halo model calculations. Note that all curves have been re-normalized to the fiducial cosmology. The thick, black, solid line shows the base template, obtained from maps generated by Sehgal et al. (2009). The black, dot-dashed line shows the modeled spectrum obtained from maps generated by applying the semi-analytic model for intracluster gas of Bode et al. (2007) to the halos identified in an N-body lightcone simulation (as described in Shaw et al. 2009). The red, solid line shows the results trom maps constructed from an adiabatic simulation produced using the Eulerian hydrodynamical code CART (Kravtsov et al. 2005), while the red, dot-dashed line shows results from maps made from the MareNostrum simulations (Zahn et al. 2010). The blue, solid line shows the predictions of the Komatsu \& Seljak (2002) halo model calculation. The histogram shows the SP'T sensitivity to the thermal SZ signal in each $\ell$-band (with arbitrary normalization) The grey band illustrates the $68 \%$ confidence interval of our theoretical uncertainty (see Figure 8 .

faint dusty, star-forming galaxies (DSFGs), the properties of which are discussed in H09. We expect the DSFG population to contain a clustered component, which will produce a power term of comparable size to the tSZ effect at $150 \mathrm{GHz}$. This clustered component would bias the measurement of power from secondary CMB anisotropies in single-frequency maps. However, we use SPT's multifrequency data to construct the DSFG-subtracted bandpowers shown in Figure 5. This frequency differencing reduces the clustered point source power to negligible levels and significantly reduces the Poisson power.

Secondary $\mathrm{CMB}$ anisotropies, consisting of a linear combination of the $\mathrm{kSZ}$ and $\mathrm{tSZ}$ effects, are detected at $2.6 \sigma$ in an analysis of the DSFG-subtracted bandpowers. Assuming there is no $\mathrm{kSZ}$ contribution, this equates to $4.2 \pm 1.5 \mu \mathrm{K}^{2}$ of tSZ power at $\ell=3000$. This is substantially lower than the $8.5 \mu \mathrm{K}^{2}$ predicted by the homogeneous kSZ and fiducial tSZ models using WMAP5 cosmological parameters. The lower than expected value of the measured SZ power may point to either lower values of $\sigma_{8}$ or an over-estimate of the modeled SZ power spectrum. Adopting a conservative prior on the uncertainty in the model predictions for the SZ amplitude, we combine the measurement of the tSZ power spectrum with CMB data on larger angular scales to improve the constraint on the CMB-derived matter power spectrum normalization, $\sigma_{8}$. This results in a decrease in the preferred value to $\sigma_{8}=0.773 \pm 0.025$. A major source of uncertainty in this analysis is due to the current theoretical uncertainty on the amplitudes and shapes of the thermal and kinetic $\mathrm{SZ}$ effect power spectra. In the absence of this theoretical uncertainty, the constraint tightens about a still lower value of $\sigma_{8}=0.746 \pm 0.017$. The tension between the predicted and measured SZ amplitudes decreases for models that predict less $\mathrm{kSZ}$ or $\mathrm{tSZ}$ power, for instance models with shorter reionization epochs or higher energy feedback in clusters. However, the tension remains at some level for all the models we considered. It is our hope that the progress in observational efforts represented by this work will lead to further work in improving theoretical models for secondary CMB anisotropies.

The SPT power spectrum results presented here use only a small fraction of the complete data set. The SPT is continuing to take data and has already observed 800 


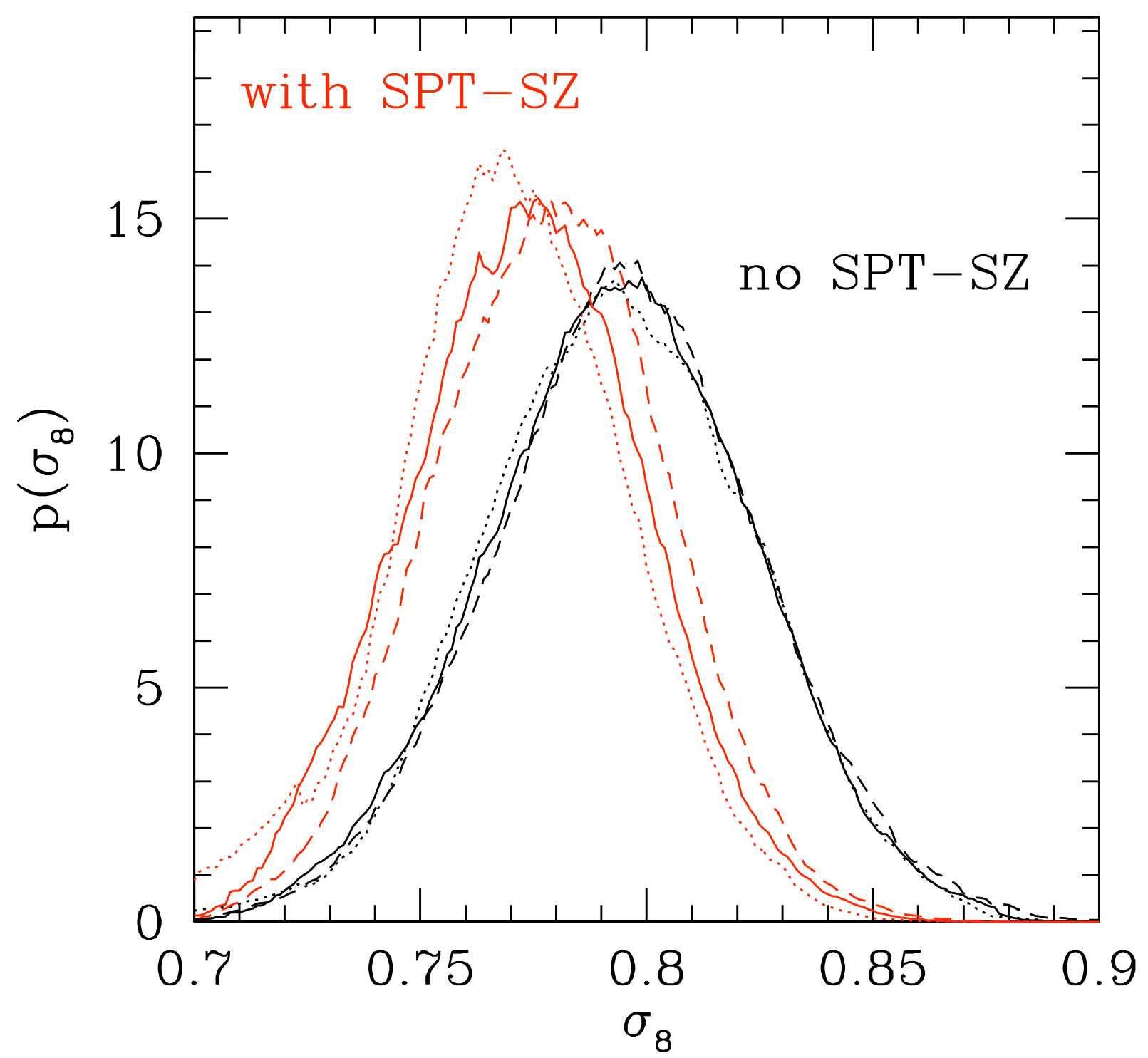

FIG. 11. - The 1D marginalized $\sigma_{8}$ constraints with and without including the SPT DSFG-subtracted bandpowers for three kSZ cases. The black lines denote the $\sigma_{8}$ constraints without SPT, while the red lines include SPT's bandpowers. Constraints with the patchy kSZ template are shown with a solid line. The results when including only the homogeneous $\mathrm{kSZ}$ model are shown with the dashed lines, and the results for no kSZ effect are shown with dotted lines. The SPT data tightens the $\sigma_{8}$ constraint in all three cases.

$\operatorname{deg}^{2}$ to similar depth at 150 and $220 \mathrm{GHz}$ to the $100 \mathrm{deg}^{2}$ used in this work, and $600 \mathrm{deg}^{2}$ of this area also has $95 \mathrm{GHz}$ coverage. The complete SPT survey is expected to cover over $2000 \mathrm{deg}^{2}$ with these three frequencies. The complete data set promises a $>10 \sigma$ detection of the tSZ power spectrum, and the addition of the third $(95 \mathrm{GHz})$ frequency band will allow us to separate the $\mathrm{kSZ}$ effect from DSFGs and the tSZ effect. Measuring the $\mathrm{kSZ}$ power spectrum will open a new window onto the reionization history of the universe. The complete SPT data set will also produce a catalog of hundreds of SZ-selected clusters, high signal-to-noise images of known clusters, a direct detection of gravitational lensing of the CMB, and the widest study to-date on the resolved and unresolved source populations at these frequencies.

The SPT team gratefully acknowledges the contributions to the design and construction of the telescope by
S. Busetti, E. Chauvin, T. Hughes, P. Huntley, and E. Nichols and his team of iron workers. We also thank the National Science Foundation (NSF) Office of Polar Programs, the United States Antarctic Program and the Raytheon Polar Services Company for their support of the project. We are grateful for professional support from the staff of the South Pole station. We thank T. M. Lanting, J. Leong, A. Loehr, W. Lu, M. Runyan, D. Schwan, M. Sharp, and C. Greer for their early contributions to the SPT project, J. Joseph and C. Vu for their contributions to the electronics and P. Ralph for his useful discussions and insights.

The South Pole Telescope is supported by the National Science Foundation through grants ANT-0638937 and ANT-0130612. Partial support is also provided by the NSF Physics Frontier Center grant PHY-0114422 to the Kavli Institute of Cosmological Physics at the University of Chicago, the Kavli Foundation and the Gordon and Betty Moore Foundation. The McGill group acknowl- 
edges funding from the National Sciences and Engineering Research Council of Canada, the Quebec Fonds de recherche sur la nature et les technologies, and the Canadian Institute for Advanced Research. The following individuals acknowledge additional support: K. Schaffer and B. Benson from a KICP Fellowship, O. Zahn from a Berkeley Center for Cosmological Physics Fellowship, J. McMahon from a Fermi Fellowship, Z. Staniszewski from a GAAN Fellowship, and A.T. Lee from the Miller Institute for Basic Research in Science, University of California Berkeley. N.W. Halverson acknowledges support from an Alfred P. Sloan Research Fellowship.

This research used resources of the National Energy Research Scientific Computing Center, which is supported by the Office of Science of the U.S. Department of Energy under Contract No. DE-AC02-05CH11231. Some of the results in this paper have been derived using the HEALPix (Górski et al. 2005) package. We acknowledge the use of the Legacy Archive for Microwave Background Data Analysis (LAMBDA). Support for LAMBDA is provided by the NASA Office of Space Science. 


\section{REFERENCES}

Bai, L., Marcillac, D., Rieke, G. H., Rieke, M. J., Tran, K., Hinz, J. L., Rudnick, G., Kelly, D. M., \& Blaylock, M. 2007, ApJ, 664,181

Bode, P., Ostriker, J. P., Weller, J., \& Shaw, L. 2007, ApJ, 663, 139

Bond, J. R., Contaldi, C. R., Pen, U.-L., Pogosyan, D., Prunet, S., Ruetalo, M. I., Wadsley, J. W., Zhang, P., Mason, B. S., Myers, S. T., Pearson, T. J., Readhead, A. C. S., Sievers, J. L., \& Udomprasert, P. S. 2005, ApJ, 626, 12

Brown, M. L., Ade, P., Bock, J., Bowden, M., Cahill, G., Castro, P. G., Church, S., Culverhouse, T., Friedman, R. B., Ganga, K., Gear, W. K., Gupta, S., Hinderks, J., Kovac, J., Lange, A. E., Leitch, E., Melhuish, S. J., Memari, Y., Murphy, J. A., Orlando, A., O'Sullivan, C., Piccirillo, L., Pryke, C., Rajguru, N., Rusholme, B., Schwarz, R., Taylor, A. N., Thompson, K. L. Turner, A. H., Wu, E. Y. S., \& Zemcov, M. 2009, ApJ, 705, 978

Carlstrom, J. E., Ade, P. A. R., Aird, K. A., Benson, B. A., Bleem, L. E., Busetti, S., Chang, C. L., Chauvin, E., Cho, H.-M., Crawford, T. M., Crites, A. T., Dobbs, M. A., Halverson, N. W., Heimsath, S., Hills, R. E., Holzapfel, W. L., Joy, M., Keisler, R., Lanting, T. M., Lee, A. T., Leitch, E. M., Leong, J., Lu, W., Lueker, M., McMahon, J. J., Meyer, S. S., Mohr, J. J., Montroy, T. E., Padin, S., Plagge, T., Pryke, C., Ruhl, J. E., Schaffer, K. K., Schwan, D., Shirokoff, E., Spieler, H. G., Staniszewski, Z., Stark, A. A., \& Vieira, J. D. 2009, submitted to PASP

Dawson, K. S., Holzapfel, W. L., Carlstrom, J. E., Joy, M., \& LaRoque, S. J. 2006, ApJ, 647, 13

de Zotti, G., Ricci, R., Mesa, D., Silva, L., Mazzotta, P., Toffolatti, L., \& González-Nuevo, J. 2005, A\&A, 431, 893

Duffy, A. R., Schaye, J., Kay, S. T., \& Dalla Vecchia, C. 2008, MNRAS, 390, L64

Dunkley, J., Komatsu, E., Nolta, M. R., Spergel, D. N., Larson, D., Hinshaw, G., Page, L., Bennett, C. L., Gold, B., Jarosik, N., Weiland, J. L., Halpern, M., Hill, R. S., Kogut, A., Limon, M., Meyer, S. S., Tucker, G. S., Wollack, E., \& Wright, E. L. 2009, ApJS, 180, 306

Finkbeiner, D. P., Davis, M., \& Schlegel, D. J. 1999, ApJ, 524, 867

Friedman, R. B., Ade, P., Bock, J., Bowden, M., Brown, M. L., Cahill, G., Castro, P. G., Church, S., Culverhouse, T., Ganga, K., Gear, W. K., Gupta, S., Hinderks, J., Kovac, J., Lange, A. E., Leitch, E., Melhuish, S. J., Memari, Y., Murphy, J. A., Orlando, A., O'Sullivan, C., Piccirillo, L., Pryke, C., Rajguru, N., Rusholme, B., Schwarz, R., Taylor, A. N., Thompson, K. L., Turner, A. H., Wu, E. Y. S., Zemcov, M., \& QUa D collaboration. 2009, ApJ, 700, L187

Glenn, J., Bock, J. J., Chattopadhyay, G., Edgington, S. F., Lange, A. E., Zmuidzinas, J., Mauskopf, P. D., Rownd, B., Yuen, L., \& Ade, P. A. 1998, Proc. SPIE, 3357, 326

Górski, K. M., Hivon, E., Banday, A. J., Wandelt, B. D., Hansen, F. K., Reinecke, M., \& Bartelmann, M. 2005, ApJ, 622, 759

Greve, T. R., Ivison, R. J., Bertoldi, F., Stevens, J. A., Dunlop, J. S., Lutz, D., \& Carilli, C. L. 2004, MNRAS, 354, 779

Haiman, Z. \& Knox, L. 2000, ApJ, 530, 124

Hall, N., Knox, L., Reichardt, C. L., Ade, P. A. R., Aird, K. A., Benson, B. B., Bleem, L. E., Carlstrom, J. E., Chang, C. L., Cho, H.-M., Crawford, T. M., T., C. A., de Haan, T., Dobbs, M. A., George, E. M., W., H. N., Holder, G. P., Holzapfel, W. L., Hrubes, J. D., Joy, M., Keisler, R., Lee, A. T., Leitch, E. M., Lueker, M., McMahon, J. J., Mehl, J., Meyer, S. S., Mohr, J. J., Montroy, T. E., Padin, S., Plagge, T., Pryke, C., E., R. J., Schaffer, K. K., Shaw, L., Shirokoff, E., Spieler, H. G., Staniszewski, Z., Stark, A. A., Switzer, E. R., Vanderlinde, K., Vieira, J. D., Williamson, R., \& Zahn, O. 2009, In Preparation Hinshaw, G., Weiland, J. L., Hill, R. S., Odegard, N., Larson, D., Bennett, C. L., Dunkley, J., Gold, B., Greason, M. R., Jarosik, N., Komatsu, E., Nolta, M. R., Page, L., Spergel, D. N.,

Wollack, E., Halpern, M., Kogut, A., Limon, M., Meyer, S. S., Tucker, G. S., \& Wright, E. L. 2009, ApJS, 180, 225

Hivon, E., Górski, K. M., Netterfield, C. B., Crill, B. P., Prunet, S., \& Hansen, F. 2002, ApJ, 567, 2
Holland, W., MacIntosh, M., Fairley, A., Kelly, D., Montgomery, D., Gostick, D., Atad-Ettedgui, E., Ellis, M., Robson, I., Hollister, M., Woodcraft, A., Ade, P., Walker, I., Irwin, K., Hilton, G., Duncan, W., Reintsema, C., Walton, A., Parkes, W., Dunare, C., Fich, M., Kycia, J., Halpern, M., Scott, D., Gibb, A., Molnar, J., Chapin, E., Bintley, D., Craig, S., Chylek, T., Jenness, T., Economou, F., \& Davis, G. 2006, in Presented at the Society of Photo-Optical Instrumentation Engineers (SPIE) Conference, Vol. 6275, Society of Photo-Optical Instrumentation Engineers (SPIE) Conference Series

Holland, W. S., Robson, E. I., Gear, W. K., Cunningham, C. R., Lightfoot, J. F., Jenness, T., Ivison, R. J., Stevens, J. A., Ade, P. A. R., Griffin, M. J., Duncan, W. D., Murphy, J. A., \& Naylor, D. A. 1999, MNRAS, 303, 659

Jenkins, A., Frenk, C. S., White, S. D. M., Colberg, J. M., Cole, S., Evrard, A. E., Couchman, H. M. P., \& Yoshida, N. 2001, MNRAS, 321, 372

Jones, W. C., Ade, P. A. R., Bock, J. J., Bond, J. R., Borrill, J., Boscaleri, A., Cabella, P., Contaldi, C. R., Crill, B. P., de Bernardis, P., De Gasperis, G., de Oliveira-Costa, A., De Troia, G., di Stefano, G., Hivon, E., Jaffe, A. H., Kisner, T. S., Lange, A. E., MacTavish, C. J., Masi, S., Mauskopf, P. D., Melchiorri, A., Montroy, T. E., Natoli, P., Netterfield, C. B., Pascale, E., Piacentini, F., Pogosyan, D., Polenta, G., Prunet, S., Ricciardi, S., Romeo, G., Ruhl, J. E., Santini, P., Tegmark, M., Veneziani, M., \& Vittorio, N. 2006, ApJ, 647, 823

Knox, L. 1999, Phys. Rev. D, 60, 103516

Knox, L., Cooray, A., Eisenstein, D., \& Haiman, Z. 2001, ApJ, 550,7

Knox, L., Holder, G. P., \& Church, S. E. 2004, ApJ, 612, 96

Komatsu, E., Dunkley, J., Nolta, M. R., Bennett, C. L., Gold, B., Hinshaw, G., Jarosik, N., Larson, D., Limon, M., Page, L., Spergel, D. N., Halpern, M., Hill, R. S., Kogut, A., Meyer, S. S., Tucker, G. S., Weiland, J. L., Wollack, E., \& Wright, E. L. 2009, ApJS, 180, 330

Komatsu, E. \& Kitayama, T. 1999, ApJ, 526, L1

Komatsu, E. \& Seljak, U. 2002, MNRAS, 336, 1256

Kravtsov, A. V., Nagai, D., \& Vikhlinin, A. A. 2005, ApJ, 625, 588

Kreysa, E., Gemuend, H., Gromke, J., Haslam, C. G., Reichertz, L., Haller, E. E., Beeman, J. W., Hansen, V., Sievers, A., \& Zylka, R. 1998, in Proc. SPIE Vol. 3357, p. 319-325, Advanced Technology MMW, Radio, and Terahertz Telescopes, Thomas G. Phillips; Ed., 319-325

Lagache, G., Bavouzet, N., Fernandez-Conde, N., Ponthieu, N., Rodet, T., Dole, H., Miville-Deschênes, M.-A., \& Puget, J.-L. 2007, ApJ, 665, L89

Lewis, A. \& Bridle, S. 2002, Phys. Rev. D, 66, 103511

Lewis, A., Challinor, A., \& Lasenby, A. 2000, ApJ, 538, 473

Lin, Y., Partridge, B., Pober, J. C., Bouchefry, K. E., Burke, S., Klein, J. N., Coish, J. W., \& Huffenberger, K. M. 2009, ApJ, 694,992

Mason, B. S., Pearson, T. J., Readhead, A. C. S., Shepherd, M. C., Sievers, J., Udomprasert, P. S., Cartwright, J. K., Farmer, A. J., Padin, S., Myers, S. T., Bond, J. R., Contaldi, C. R., Pen, U., Prunet, S., Pogosyan, D., Carlstrom, J. E., Kovac, J., Leitch, E. M., Pryke, C., Halverson, N. W., Holzapfel, W. L., Altamirano, P., Bronfman, L., Casassus, S., May, J., \& Joy, M. 2003, ApJ, 591, 540

Negrello, M., Perrotta, F., González-Nuevo, J., Silva, L., de Zotti, G., Granato, G. L., Baccigalupi, C., \& Danese, L. 2007, MNRAS, 377, 1557

Nolta, M. R., Dunkley, J., Hill, R. S., Hinshaw, G., Komatsu, E., Larson, D., Page, L., Spergel, D. N., Bennett, C. L., Gold, B., Jarosik, N., Odegard, N., Weiland, J. L., Wollack, E., Halpern, M., Kogut, A., Limon, M., Meyer, S. S., Tucker, G. S., \& Wright, E. L. 2009, ApJS, 180, 296 
Padin, S., Staniszewski, Z., Keisler, R., Joy, M., Stark, A. A., Ade, P. A. R., Aird, K. A., Benson, B. A., Bleem, L. E., Carlstrom, J. E., Chang, C. L., Crawford, T. M., Crites, A. T., Dobbs, M. A., Halverson, N. W., Heimsath, S., Hills, R. E., Holzapfel, W. L., Lawrie, C., Lee, A. T., Leitch, E. M., Leong, J., Lu, W., Lueker, M., McMahon, J. J., Meyer, S. S., Mohr, J. J., Montroy, T. E., Plagge, T., Pryke, C., Ruhl, J. E., Schaffer, K. K., Shirokoff, E., Spieler, H. G., \& Vieira, J. D. 2008, Appl. Opt., 47, 4418

Pascale, E., Ade, P. A. R., Bock, J. J., Chapin, E. L., Chung, J., Devlin, M. J., Dicker, S., Griffin, M., Gundersen, J. O., Halpern, M., Hargrave, P. C., Hughes, D. H., Klein, J., MacTavish, C. J., Marsden, G., Martin, P. G., Martin, T. G., Mauskopf, P., Netterfield, C. B., Olmi, L., Patanchon, G., Rex, M., Scott, D., Semisch, C., Thomas, N., Truch, M. D. P., Tucker, C., Tucker, G. S., Viero, M. P., \& Wiebe, D. V. 2008, ApJ, 681, 400

Polenta, G., Marinucci, D., Balbi, A., de Bernardis, P., Hivon, E., Masi, S., Natoli, P., \& Vittorio, N. 2005, Journal of Cosmology and Astro-Particle Physics, 11, 1

Reichardt, C. L., Ade, P. A. R., Bock, J. J., Bond, J. R., Brevik, J. A., Contaldi, C. R., Daub, M. D., Dempsey, J. T., Goldstein, J. H., Holzapfel, W. L., Kuo, C. L., Lange, A. E., Lueker, M., Newcomb, M., Peterson, J. B., Ruhl, J., Runyan, M. C., \& Staniszewski, Z. 2009a, ApJ, 694, 1200

Reichardt, C. L., Zahn, O., Ade, P. A. R., Basu, K., Bender, A. N., Bertoldi, F., Cho, H. ., Chon, G., Dobbs, M., Ferrusca, D., Halverson, N. W., Holzapfel, W. L., Horellou, C., Johansson, D., Johnson, B. R., Kennedy, J., Kneissl, R., Lanting, T., Lee, A. T., Lueker, M., Mehl, J., Menten, K. M., Nord, M., Pacaud, F., Richards, P. L., Schaaf, R., Schwan, D., Spieler, H., Weiss, A., \& Westbrook, B. 2009b, ApJ, 701, 1958

Righi, M., Hernández-Monteagudo, C., \& Sunyaev, R. A. 2008, A\&A, 478, 685

Ruhl, J., Ade, P. A. R., Carlstrom, J. E., Cho, H.-M., Crawford, T., Dobbs, M., Greer, C. H., Halverson, N. W., Holzapfel, W. L., Lanting, T. M., Lee, A. T., Leitch, E. M., Leong, J., Lu, W., Lueker, M., Mehl, J., Meyer, S. S., Mohr, J. J., Padin, S., Plagge, T., Pryke, C., Runyan, M. C., Schwan, D., Sharp, M. K., Spieler, H., Staniszewski, Z., \& Stark, A. A. 2004, in Proc. SPIE, Vol. 5498, Millimeter and Submillimeter Detectors for Astronomy II, ed. J. Zmuidzinas, W. S. Holland, \& S. Withington (Bellingham: SPIE Optical Engineering Press), $11-29$

Sayers, J., Golwala, S. R., Rossinot, P., Ade, P. A. R., Aguirre, J. E., Bock, J. J., Edgington, S. F., Glenn, J., Goldin, A., Haig, D., Lange, A. E., Laurent, G. T., Mauskopf, P. D., \& Nguyen, H. T. 2009, ApJ, 690, 1597

Scott, K. S., Austermann, J. E., Perera, T. A., Wilson, G. W., Aretxaga, I., Bock, J. J., Hughes, D. H., Kang, Y., Kim, S., Mauskopf, P. D., Sanders, D. B., Scoville, N., \& Yun, M. S. 2008, MNRAS, 385, 2225

Sehgal, N., Bode, P., Das, S., Hernandez-Monteagudo, C., Huffenberger, K., Lin, Y.-T., Ostriker, J. P., \& Trac, H. 2009, submitted to ApJ, astro-ph/0908.0540

Sharp, M. K., Marrone, D. P., Carlstrom, J. E., Culverhouse, T., Greer, C., Hawkins, D., Hennessy, R., Joy, M., Lamb, J. W., Leitch, E. M., Loh, M., Miller, A., Mroczkowski, T., Muchovej, S., Pryke, C., \& Woody, D. 2009, submitted to ApJ, astro-ph/0901.4342
Shaw, L. D., Zahn, O., Holder, G. P., \& Doré, O. 2009, ApJ, 702, 368

Sievers, J. L., Mason, B. S., Weintraub, L., Achermann, C., Altamirano, P., Bond, J. R., Bronfman, L., Bustos, R., Contaldi, C., Dickinson, C., Jones, M. E., May, J., Myers, S. T., Oyarce, N., Padin, S., Pearson, T. J., Pospieszalski, M., Readhead, A. C. S., Reeves, R., Shepherd, M. C., Taylor,

A. C., \& Torres, S. 2009, submitted to ApJ, astro-ph/0901.4540 Silk, J. 1968, ApJ, 151, 459

Siringo, G., Kreysa, E., Kovács, A., Schuller, F., Weiß, A., Esch, W., Gemünd, H., Jethava, N., Lundershausen, G., Colin, A., Güsten, R., Menten, K. M., Beelen, A., Bertoldi, F., Beeman, J. W., \& Haller, E. E. 2009, A\&A, 497, 945

Staniszewski, Z., Ade, P. A. R., Aird, K. A., Benson, B. A., Bleem, L. E., Carlstrom, J. E., Chang, C. L., Cho, H.-M., Crawford, T. M., Crites, A. T., de Haan, T., Dobbs, M. A., Halverson, N. W., Holder, G. P., Holzapfel, W. L., Hrubes, J. D., Joy, M., Keisler, R., Lanting, T. M., Lee, A. T., Leitch, E. M., Loehr, A., Lueker, M., McMahon, J. J., Mehl, J., Meyer, S. S., Mohr, J. J., Montroy, T. E., Ngeow, C.-C., Padin, S., Plagge, T., Pryke, C., Reichardt, C. L., Ruhl, J. E., Schaffer, K. K., Shaw, L., Shirokoff, E., Spieler, H. G., Stalder, B. Stark, A. A., Vanderlinde, K., Vieira, J. D., Zahn, O., \& Zenteno, A. 2009, ApJ, 701, 32

Sunyaev, R. A. \& Zeldovich, Y. B. 1972, Comments on Astrophysics and Space Physics, 4, 173

-. 1980, MNRAS, 190, 413

Tristram, M., Macías-Pérez, J. F., Renault, C., \& Santos, D. 2005, MNRAS, 358, 833

Vieira, J. D., Crawford, T. M., Switzer, E. R., Ade, P. A. R., Aird, K. A., Ashby, M. L. N., Benson, B. A., Bleem, L. E., Brodwin, M., Carlstrom, J. E., Chang, C. L., Cho, H., Crites, A. T., de Haan, T., Dobbs, M. A., Everett, W., George, E. M., Gladders, M., Hall, N. R., Halverson, N. W., High, F. W., Holder, G. P., Holzapfel, W. L., Hrubes, J. D., Joy, M., Keisler, R., Knox, L., Lee, A. T., Leitch, E. M., Lueker, M., Marrone, D. P., McIntyre, V., McMahon, J. J., Mehl, J., Meyer, S. S., Mohr, J. J., Montroy, T. E., Padin, S., Plagge, T., Pryke, C., Reichardt, C. L., Ruhl, J. E., Schaffer, K. K., Shaw, L., Shirokoff, E., Spieler, H. G., Stalder, B., Staniszewski, Z. Stark, A. A., Vanderlinde, K., Walsh, W., Williamson, R., Yang, Y., Zahn, O., \& Zenteno, A. 2009, submitted to ApJ, astro-ph/0912.2338

Viero, M. P., Ade, P. A. R., Bock, J. J., Chapin, E. L., Devlin, M. J., Griffin, M., Gundersen, J. O., Halpern, M., Hargrave, P. C., Hughes, D. H., Klein, J., MacTavish, C. J., Marsden, G., Martin, P. G., Mauskopf, P., Moncelsi, L., Negrello, M., Netterfield, C. B., Olmi, L., Pascale, E., Patanchon, G., Rex, M., Scott, D., Semisch, C., Thomas, N., Truch, M. D. P., Tucker, C., Tucker, G. S., \& Wiebe, D. V. 2009, accepted by ApJ, astro-ph/0904.1200

Zahn, O., Gottloeber, S., \& Yepes, G. 2010, in prep.

Zahn, O., Zaldarriaga, M., Hernquist, L., \& McQuinn, M. 2005, ApJ, 630, 657

\section{APPENDIX}

\section{BANDPOWER COVARIANCE MATRIX ESTIMATION}

The bandpower covariance matrix includes both signal and noise contributions. The signal covariance is calculated from simulations. The noise covariance is estimated from the data. We calculate the variance of the mean power spectrum using the variance of cross-spectra between independent real maps. With 300 independent observations of the same field, these maps are sufficient to generate an accurate estimate of the covariance matrix. Several details of our approach are motivated by the analytical treatments of Tristram et al. (2005) and Polenta et al. (2005), and we first review the analytic estimate of the covariance matrix before discussing the estimator. 


\section{Analytical Considerations}

Following Tristram et al. (2005), we represent the expected covariance between two cross spectra as $\Xi$ :

$$
\begin{aligned}
\Xi_{\ell \ell^{\prime}}^{A B, C D} & \equiv\left\langle\left(\widehat{D}_{\ell}^{A B}-\left\langle\widehat{D}_{\ell}^{A B}\right\rangle\right)\left(\widehat{D}_{\ell^{\prime}}^{C D}-\left\langle\widehat{D}_{\ell^{\prime}}^{C D}\right\rangle\right)\right\rangle \\
& =\left(K[\mathbf{W}]^{-1}\right)_{b^{\prime \prime} b}\left(\left\langle D_{b^{\prime \prime}}^{A B} D_{b^{\prime \prime \prime}}^{C D}\right\rangle-\left\langle D_{b^{\prime \prime}}^{A B}\right\rangle\left\langle D_{b^{\prime \prime \prime}}^{C D}\right\rangle\right)\left(K[\mathbf{W}]^{-1}\right)_{b^{\prime \prime \prime} b^{\prime}} .
\end{aligned}
$$

Our goal is to express this covariance in terms of the noise and signal in the maps, and to compute the magnitude of the diagonal elements, as well as the correlation between bandpowers. As the first step, the central term can be rewritten as,

$$
\begin{aligned}
\left\langle D_{b}^{A B} D_{b^{\prime}}^{C D}\right\rangle-\left\langle D_{b}^{A B}\right\rangle\left\langle D_{b^{\prime}}^{C D}\right\rangle & =P_{b k} P_{b^{\prime} k^{\prime}} \frac{1}{(2 \pi)^{2}} \int d \theta_{k} d \theta_{k^{\prime}}\left(\left\langle\widetilde{m}_{\mathbf{k}}^{A} \widetilde{m}_{\mathbf{k}}^{B *} \widetilde{m}_{\mathbf{k}^{\prime}}^{C} \widetilde{m}_{\mathbf{k}^{\prime}}^{D *}\right\rangle-\left\langle\widetilde{m}_{\mathbf{k}}^{A} \widetilde{m}_{\mathbf{k}}^{B *}\right\rangle\left\langle\widetilde{m}_{\mathbf{k}^{\prime}}^{C} \widetilde{m}_{\mathbf{k}^{\prime}}^{D *}\right\rangle\right) \\
& =P_{b k} P_{b^{\prime} k^{\prime}} \frac{1}{(2 \pi)^{2}} \int d \theta_{k} d \theta_{k^{\prime}}\left(\left\langle\widetilde{m}_{\mathbf{k}}^{A} \widetilde{m}_{\mathbf{k}^{\prime}}^{C}\right\rangle\left\langle\widetilde{m}_{\mathbf{k}}^{B *} \widetilde{m}_{\mathbf{k}^{\prime}}^{D *}\right\rangle+\left\langle\widetilde{m}_{\mathbf{k}}^{A} \widetilde{m}_{\mathbf{k}^{\prime}}^{D *}\right\rangle\left\langle\widetilde{m}_{\mathbf{k}}^{B *} \widetilde{m}_{\mathbf{k}^{\mathbf{k}^{\prime}}}^{C}\right\rangle\right) .
\end{aligned}
$$

To simplify equation A3, Tristram et al. (2005) and Polenta et al. (2005) make the following assumptions:

1. Fluctuations in the map, i.e., from CMB anisotropies, confusion limited point sources and noise, are well described by a Gaussian random field.

2. The beams and filtering applied to the data are isotropic; $E_{\mathbf{k}} \equiv G_{\mathbf{k}} B_{\mathbf{k}}$ depends only on $|\mathbf{k}|$.

3. The instrumental noise is isotropic.

4. The power spectrum, $C_{k}$, is smoothly varying with $k$, and changes little over scales comparable to the width of the mode coupling matrix.

Using the assumption that $C_{k}$ and $\left|E_{\mathbf{k}}\right|$ do not vary much over small changes in $\mathbf{k}$, these products become:

$$
\left\langle\widetilde{m}_{\mathbf{k}}^{A} \widetilde{m}_{\mathbf{k}^{\prime}}^{B *}\right\rangle=\sum_{\mathbf{k}^{\prime \prime} \mathbf{k}^{\prime \prime \prime}} \widetilde{\mathbf{W}}_{\mathbf{k}-\mathbf{k}^{\prime \prime}} \widetilde{\mathbf{W}}_{\mathbf{k}^{\prime}-\mathbf{k}^{\prime \prime \prime}}^{*} E_{\mathbf{k}^{\prime \prime}} E_{\mathbf{k}^{\prime \prime \prime}}^{*}\left\langle a_{\mathbf{k}^{\prime \prime}}^{A} a_{\mathbf{k}^{\prime \prime \prime}}^{B *}\right\rangle=\sum_{\mathbf{k}^{\prime \prime}} \widetilde{\mathbf{W}}_{\mathbf{k}-\mathbf{k}^{\prime \prime}} \widetilde{\mathbf{W}}_{\mathbf{k}^{\prime \prime}-\mathbf{k}^{\prime}}\left|E_{\mathbf{k}^{\prime \prime}}\right|^{2} C_{k^{\prime \prime}}^{A B} \approx \widetilde{\mathbf{W}^{2}} \mathbf{k}-\mathbf{k}^{\prime}\left|E_{\mathbf{k}}\right|^{2} C_{k}^{A B}
$$

Here $C_{k}^{A B}$ is shorthand for $C_{k}+\frac{N_{k}^{A}}{B_{k}^{2}} \delta_{A B}$, the expected cross spectrum between two unfiltered, perfectly beamcorrected - though noisy - maps. The additional term is the noise bias that exists in the map auto-spectrum. Assuming isotropic beams and filtering, we can combine equations $\mathrm{A} 3$ and $\mathrm{A} 4$ to obtain a relatively simple expression:

$$
\begin{aligned}
\left\langle D_{b}^{A B} D_{b^{\prime}}^{C D}\right\rangle-\left\langle D_{b}^{A B}\right\rangle\left\langle D_{b^{\prime}}^{C D}\right\rangle & =P_{b k} P_{b^{\prime} k^{\prime}} \frac{1}{(2 \pi)^{2}} \int d \theta_{k} d \theta_{k^{\prime}}\left|\widetilde{\mathbf{W}^{2}}\right|^{2}\left|E_{k}\right|^{2}\left|E_{k^{\prime}}\right|^{2}\left(C_{k}^{A B} C_{k^{\prime}}^{C D}+C_{k}^{A D} C_{k^{\prime}}^{B C}\right) \\
& =P_{b k} P_{b^{\prime} k^{\prime}} E_{k}^{2} E_{k^{\prime}}^{2} M\left[\mathbf{W}^{2}\right]_{k k^{\prime}}\left(C_{k}^{A C} C_{k^{\prime}}^{B D}+C_{k}^{A D} C_{k^{\prime}}^{B C}\right) .
\end{aligned}
$$

Combining equations A3 and A5 yields:

$$
\Xi_{b b^{\prime}}^{A B, C D}=\left(K[\mathbf{W}]^{-1}\right)_{b^{(2) b}}\left[P_{b^{(2)} k} P_{b^{(3)} k^{\prime}} M\left[\mathbf{W}^{2}\right]_{k k^{\prime}} E_{k}^{2} E_{k^{\prime}}^{2}\left(C_{k}^{A C} C_{k^{\prime}}^{B D}+C_{k}^{A D} C_{k^{\prime}}^{B C}\right)\right]\left(K[\mathbf{W}]^{-1}\right)_{b^{(3)} b^{\prime}} .
$$

To obtain a simplified expression for the magnitude of the diagonal elements of the covariance matrix, one typically assumes the mode-coupling matrix is nearly diagonal: $M_{k k^{\prime}}[\mathbf{W}] \approx w_{2} \delta_{k k^{\prime}}$. In this approximation $\left(K[\mathbf{W}]^{-1}\right)_{b b^{\prime}} \approx$ $w_{2}^{-1} E_{b}^{-2} \delta_{b b^{\prime}}$ and $M_{k k^{\prime}}\left[\mathbf{W}^{2}\right] \approx w_{4} \delta_{k k^{\prime}}$ and thus the covariance of any two cross spectra is:

$$
\begin{aligned}
\Xi_{b b^{\prime}}^{A B, C D} & \approx \frac{w_{4}}{N_{b} w_{2}^{2}}\left(\frac{\ell_{\mathrm{eff}, b}\left(\ell_{\mathrm{eff}, b}+1\right)}{2 \pi}\right)^{2}\left(C_{b}^{A C} C_{b}^{B D}+C_{b}^{A D} C_{b}^{B C}\right) \delta_{b b^{\prime}} \\
& =\frac{\left\langle\widehat{D}_{b}^{A C}\right\rangle\left\langle\widehat{D}_{b}^{B D}\right\rangle+\left\langle\widehat{D}_{b}^{A D}\right\rangle\left\langle\widehat{D}_{b}^{B C}\right\rangle}{\nu_{b}} \delta_{b b^{\prime}},
\end{aligned}
$$

where $\nu_{b}$ is the effective number of independent k-modes in each $\ell$-band. For isotropic filtering, $\nu_{b}^{\text {iso }}=\frac{N_{b} w_{2}^{2}}{w_{4}}$.

One subtlety in estimating the covariance is the fact that although the noise in each map is independent, each map has the same sky coverage. Hence the signal in all maps is correlated. The correct estimator must take this correlation into account. Under the simplifying assumption that all maps are statistically equivalent, the correlation between two cross spectra depends only on whether the spectra have a map in common (e.g. the cross spectrum $D_{b}^{12}$ is more strongly correlated to the spectrum $D_{b}^{23}$ than $D_{b}^{34}$ ). Comparing the covariance of two cross-spectra taken from 4 different observations:

$$
\left.\Xi_{b b}^{A B, C D}\right|_{A \neq B \neq C \neq D}=\frac{2}{\nu_{b}} C_{b}^{2},
$$


to the covariance of a pair of cross-spectra with a common map,

$$
\left.\Xi_{b b}^{A B, B C}\right|_{A \neq B \neq C}=\frac{1}{\nu_{b}}\left(2 C_{b}^{2}+\frac{N_{b}}{B_{b}^{2}} C_{b}\right),
$$

to the covariance of a pair of cross-spectra with two maps in common,

$$
\left.\Xi_{b b}^{A B, A B}\right|_{A \neq B}=\frac{1}{\nu_{b}}\left(2 C_{b}^{2}+2 \frac{N_{b}}{B_{b}^{2}} C_{b}+\frac{N_{b}^{2}}{B_{b}^{4}}\right) .
$$

The degree of correlation is also $\ell$ dependent since it depends on the relative signal vs. noise power in the maps. In the high- $\ell$ regime, where noise dominates the power in an individual map, all cross spectra are nearly independent. Conversely all cross-spectra are nearly completely correlated at low- $\ell$, where the primary CMB anisotropy overwhelms the noise.

Given the assumption of statistical equivalence, we can then compute the expected variance of the mean spectrum based on these variance estimates. This is equal to the correlation between any particular cross-spectrum and the mean,

$$
\Xi_{b b}^{\text {mean,mean }}=\left.\Xi_{b b}^{\text {mean }, A B}\right|_{A \neq B} \approx \frac{1}{\nu_{b}}\left(2 C_{b}^{2}+4 C_{b} \frac{N_{b}}{n_{o b s} B_{b}^{2}}+2 \frac{N_{b}^{2}}{n_{o b s}^{2} B_{b}^{4}}\right) .
$$

This estimate of the variation of the mean spectrum agrees with the uncertainty estimates given in Polenta et al. (2005).

It should be noted that the noise and filtering of the SPT data are anisotropic. Equation A7 can therefore be used only as a guideline. In the case of anisotropic filtering or anisotropic beams, the number of independent modes per bin will be typically smaller than $\nu_{b}^{\text {iso }}$, since anisotropic filtering will weight different k-space modes unevenly. For example, the k-space mask completely eliminates all modes with $k_{x}<1200$. The variance in each $\ell$-bin increases with fewer independent modes. However, even if we account for the effective number of independent modes in an $\ell$-bin, equation A7 does not account for the anisotropic nature of the atmospheric noise contribution.

\section{The Empirical Covariance Estimator}

The existing analytic treatments are not directly applicable to the SPT data due to anisotropies in the noise and filters. By the nature of SPT's scan strategy, atmospheric fluctuations preferentially contaminate low $k_{x}$ modes. Likewise the filters intended to remove these fluctuations preferentially remove low $k_{x}$ modes. Instead, we have designed an empirical estimator which reproduces the analytical results when applied to isotropic data, while accurately accounting for the increased uncertainty due to the noise and filtering anisotropies in the actual data.

The noise covariance matrix estimate is divided into two parts, a signal contribution obtained from the Monte Carlo simulations described in 3.2 .3 and a noise contribution obtained from real single-observation maps:

$$
\mathbf{C}_{b b^{\prime}}=\mathbf{C}_{b b^{\prime}}^{\mathrm{MC}, \mathrm{s}}+\mathbf{C}_{b b^{\prime}}^{\text {data }} .
$$

The signal contribution is straightforward to estimate with an approach similar to the MASTER power spectrum error estimator. We use the signal only simulations to obtain an empirical estimate of the sample variance:

$$
\mathrm{C}_{b b^{\prime}}^{\mathrm{MC}, \mathrm{s}}=\overline{\Delta \widehat{D}_{b}^{\mathrm{MC}, \mathrm{s}} \Delta \widehat{D}_{b^{\prime}}^{\mathrm{MC}, \mathrm{s}}} .
$$

Note that here $\Delta x \equiv x-\bar{x}$ is defined with respect to the sample mean. Since the simulations include only CMB realizations and point sources in the confusion limit, the simulated signals are essentially Gaussian. Therefore we expect the usual sample variance contribution:

$$
\left\langle\mathbf{C}_{b b^{\prime},(\mathrm{MC}, \mathrm{s})}\right\rangle=\frac{2 C_{b}^{\text {theory }}}{\nu_{b}} .
$$

As before, $\nu_{b}$ is the effective number of independent Fourier-modes in each $\ell$-band.

The noise contribution is computed from the cross spectra of single-observation maps. We use the following estimator for the noise contribution:

$$
\mathbf{C}_{b b^{\prime}}^{\mathrm{data}} \equiv \frac{2 f\left(n_{\mathrm{obs}}\right)}{n_{\mathrm{obs}}^{4}} \sum_{\lambda} \sum_{\alpha \neq \lambda}\left(\Delta \widehat{D}_{b}^{\lambda \alpha} \Delta \widehat{D}_{b^{\prime}}^{\lambda \alpha}+2\left[\sum_{\beta \neq \lambda, \alpha} \Delta \widehat{D}_{b}^{\lambda \alpha} \Delta \widehat{D}_{b^{\prime}}^{\lambda \beta}\right]\right) .
$$

Here $f\left(n_{\text {obs }}\right)$ is a correction due to the finite number of realizations. In the limit of many observations this function asymptotes to unity; we use 300 observations so this term can be ignored. The first term can be identified as the sample variance of the cross spectra. The second term accounts for the additional correlations between cross-spectra with a common map.

We can now calculate the expectation value for the noise component of the covariance estimator defined in A15. 


$$
\left\langle\mathbf{C}_{b b}^{\text {data }}\right\rangle \approx \frac{2}{n_{o b s}^{2}} \Xi_{b b}^{\lambda \alpha, \lambda \alpha}+\frac{4}{n_{o b s}} \Xi_{b b}^{\lambda \alpha, \lambda \beta}-\left(\frac{4}{n_{o b s}}+\frac{2}{n_{o b s}^{2}}\right) \Xi^{\text {mean,mean }}=\frac{1}{\nu_{b}}\left(4 C_{b} \frac{N_{b}}{n_{o b s} B_{b}^{2}}+2 \frac{N_{b}^{2}}{n_{o b s}^{2} B_{b}^{4}}\right) .
$$

This is combined with the signal, or cosmic variance, component in equation A13 to get the expectation value of the estimator:

$$
\left\langle\mathbf{C}_{b b}\right\rangle \approx \frac{1}{\nu_{b}}\left[2 C_{b}^{\text {theory }^{2}}+4 C_{b} \frac{N_{b}}{n_{o b s} B_{b}^{2}}+2 \frac{N_{b}^{2}}{n_{o b s}^{2} B_{b}^{4}}\right] .
$$

This agrees with the analytic estimate (equation A11) for the variance of the mean.

\section{Multifrequency Cross Covariances}

A multifrequency data set requires an estimate of both the covariance of the each individual set of bandpowers (i.e., both the single-frequency bandpowers, and the cross-frequency bandpowers) and the cross-covariance between these sets. We naturally expect signal correlations between different sets of bandpower due to the fact that all three sets of bandpowers reported here are derived from the same patch of sky. However we also expect noise correlations between the $150 \mathrm{GHz} \times 220 \mathrm{GHz}$ cross spectrum bandpowers and each set of single-frequency bandpowers since the noise uncertainty in the cross-spectrum is entirely due to noise in the $150 \mathrm{GHz}$ and $220 \mathrm{GHz}$ data. Thus we compute the cross-covariance matrices, $\mathbf{C}_{b b^{\prime}}{ }^{(i, j)}$, where $\mathrm{i}$ and $\mathrm{j}$ denote one of the three sets of bandpowers: $150 \mathrm{GHz}, 220 \mathrm{GHz}$ and $150 \mathrm{GHz} \times 220 \mathrm{GHz}$ :

$$
\mathbf{C}_{b b^{\prime}}{ }^{(i, j)}=\overline{\Delta \widehat{D}_{b}^{\mathrm{MC}, \mathrm{s}^{(i)}} \Delta \widehat{D}_{b^{\prime}}^{\mathrm{MC}, \mathrm{s}^{(j)}}}+\frac{2 f\left(n_{o b s}\right)}{n_{m}^{4}} \sum_{\lambda} \sum_{\alpha \neq \lambda}\left(\Delta \widehat{D}_{b}^{\lambda \alpha}{ }^{(i)} \Delta \widehat{D}_{b^{\prime}}^{\lambda \alpha}{ }^{(j)}+2\left[\sum_{\beta \neq \lambda, \alpha} \Delta \widehat{D}_{b}^{\lambda \alpha}{ }^{(i)} \Delta \widehat{D}_{b^{\prime}}^{\lambda \beta^{(j)}}\right]\right) .
$$

\section{Treatment of Off-diagonal Elements}

Given the finite number of simulations and data maps, we expect some statistical uncertainty in the covariance estimate, particularly in the off-diagonal elements. Such uncertainty is not unique to the estimation technique described here, rather it is expected for any covariance estimate which is computed from a finite number of realizations. We expect the covariance estimates to be Wishart distributed, with $n_{o b s}=300$ degrees of freedom. A given covariance element, $\mathbf{C}_{i j}$ has a statistical variance of:

$$
\left\langle\left(\mathbf{C}_{i j}-\left\langle\mathbf{C}_{i j}\right\rangle\right)^{2}\right\rangle=\frac{\mathbf{C}_{i j}^{2}+\mathbf{C}_{i i} \mathbf{C}_{j j}}{n_{o b s}}
$$

For diagonal elements we expect a standard deviation of $\sqrt{2 / n_{o b s}}=1 / \sqrt{150}=8.1 \%$. In addition, there is a statistical uncertainty on the apparent correlation between two bins. If we assume that the true correlation between bins is small, then the standard deviation of the apparent correlation between two bins is $\sqrt{1 / n_{\text {obs }}}$ or $5.7 \%$. For the choice of bin-size, the statistical error on the correlation of any two bins is much larger than the expected correlation (i.e. the fractional error on the apparent correlation estimates is greater than $100 \%$ even for adjacent bins). For bins that are widely separated, these false bin-bin correlations may skew model fitting. Therefore we "condition" the published covariance matrices in order to reduce this statistical uncertainty on the covariance matrices.

From equation A5, we see that the shape of the correlation matrix (i.e. the relative size of the on-diagonal to off-diagonal covariance elements as a function of bin separation) is determined by the apodization window through the quadratic mode-coupling matrix, $M[\mathbf{W}]_{k k}$. For the $\ell$ range considered in this work, the off-diagonal elements of this matrix depend only on the distance from the diagonal, $\left|k-k^{\prime}\right|$. Therefore we condition the estimated covariance matrix, $\widehat{\mathbf{C}}_{k k^{\prime}}$ by first computing the corresponding correlation matrix, and then averaging all off-diagonal elements of a fixed separation from the diagonal:

$$
\mathbf{C}_{k k^{\prime}}^{\prime}=\frac{\sum_{k_{1}-k_{2}=k-k^{\prime}} \frac{\widehat{\mathbf{C}}_{k_{1} k_{2}}}{\sqrt{\widehat{\mathbf{C}}_{k_{1} k_{1}} \widehat{\mathbf{C}}_{k_{2} k_{2}}}}}{\sum_{k_{1}-k_{2}=k-k^{\prime}} 1} .
$$

The bandpowers reported in Tables 1 and 2 are obtained by first computing power spectra and covariance matrices for a bin-width of $\Delta \ell=100$ with a total of 80 preliminary bins. This covariance matrix was then conditioned according to equation A20 before averaging the bandpowers and covariance matrix into the final bands.

Equation A5 is based on the assumption that the filtering is isotropic. In order to test the validity of this equation for the anisotropic filtering, we perform 10000 simple Monte Carlo simulations. In each simulation a white noise realization is subjected to a simplified, though similarly anisotropic, version of the filtering scheme. The variance of the resultant spectra is computed and compared to equation A5. Though the apparent correlations between all bins exhibit the expected $1 \%$ scatter, the correlations between neighboring bins are consistent with equation A5. 